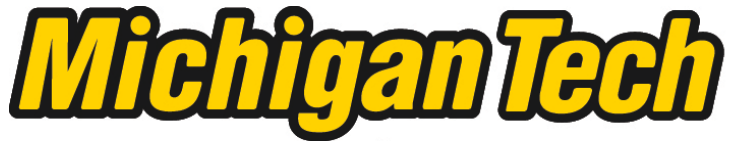 \\ Michigan Technological University Create the Future Digital Commons @ Michigan Tech
}

2014

\section{AN ASSESSMENT OF FLOW REGIME MAPS AND A NUMERICAL HEAT TRANSFER CORRELATION FOR THE STRATIFIED/ANNULAR REGIME OF SHEAR-DRIVEN INTERNAL CONDENSING FLOWS}

FNU Nikhil Shankar

Michigan Technological University

Follow this and additional works at: https://digitalcommons.mtu.edu/etds

Part of the Applied Mechanics Commons

Copyright 2014 FNU Nikhil Shankar

Recommended Citation

Nikhil Shankar, FNU, "AN ASSESSMENT OF FLOW REGIME MAPS AND A NUMERICAL HEAT TRANSFER CORRELATION FOR THE STRATIFIED/ANNULAR REGIME OF SHEAR-DRIVEN INTERNAL CONDENSING FLOWS", Master's Thesis, Michigan Technological University, 2014.

https://doi.org/10.37099/mtu.dc.etds/798

Follow this and additional works at: https://digitalcommons.mtu.edu/etds

Part of the Applied Mechanics Commons 


\title{
AN ASSESSMENT OF FLOW REGIME MAPS AND A NUMERICAL HEAT TRANSFER CORRELATION FOR THE STRATIFIED/ANNULAR REGIME OF SHEAR-DRIVEN INTERNAL CONDENSING FLOWS
}

\author{
By \\ Nikhil Shankar

\begin{abstract}
A THESIS
Submitted in partial fulfillment of the requirements for the degree of

MASTER OF SCIENCE

In Mechanical Engineering
\end{abstract} \\ MICHIGAN TECHNOLOGICAL UNIVERSITY \\ 2014
}

(C) 2014 Nikhil Shankar 
This thesis has been approved in partial fulfillment of the requirements for the Degree of MASTER OF SCIENCE in Mechanical Engineering.

Department of Mechanical Engineering-Engineering Mechanics

\author{
Thesis Advisor: $\quad$ Dr. Amitabh Narain \\ Committee Member: $\quad$ Dr. Sunil S. Mehendale \\ Committee Member: $\quad$ Dr. VC Rao Komaravolu \\ Department Chair: $\quad$ Dr. William W. Predebon
}




\section{Table of Contents}

List of Figures..............................................................................................................................vi

Acknowledgments.......................................................................................................................ix

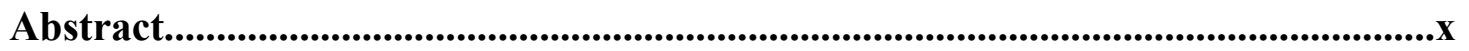

1 Background and Introduction ..............................................................

1.1 Need for Research in Flow Condensers......................................................

1.2 Design Related Issues with Internal Condensing Flows..............................2

2 Predictive Capabilities of Flow Condensers..............................................

2.1 Computational Tools Used in this Study....................................................

2.2 Theoretical Model for Internal Condensing Flows.....................................

2.3 Two Dimensional Governing Equations for Condensing Flows.................12

2.4 Two Dimensional Steady Condensing Flow Solver....................................17

2.5 1D Approximation for Complete 2D Governing Equations.......................18

2.6 Quasi-1D Steady Condensing Flow Simulation Tool................................21 
3 Flow Regimes in Shear Driven Internal Condensing Flows

.23

3.1 Introduction to Flow Morphology and the Importance of Flow Regimes in Shear-Driven Internal Condensing Flows........................................... 23

3.2 Association of Heat Transfer Correlations with Studies on Flow Regimes in Internal Condensing Flows...........................................................2

3.3 Analysis of Flow Regime Maps in Literature...........................................33

3.3.1 Flow Regime Map of Taitel and Dukler......................................34

3.3.2 Flow Regime Map of Baker...................................................4

3.4 Relationship of Vapor Quality to Physical Distance in a Condenser.........47

3.5 A Quantitative Flow Regime Map in the $\left\{\frac{\mathrm{Ja}}{P r_{1}}, R e_{i n}, \hat{x}\right\}$ space..................50

4 Heat Transfer in the Stratified/Annular Flow Regime..............................55

4.1 Engineering Heat Transfer Correlations in Literature..............................55

4.1.1 Heat Transfer Correlation of Kim and Mudawar..........................57

4.1.2 Heat Transfer Correlation of Koyama et al................................59

4.1.3 Heat Transfer Correlation of Cavallini and Zecchin.....................60

4.2 A Computationally Derived Heat Transfer Correlation...........................62

4.2.1 Correlation as a Function of Distance.........................................64

4.2.2 Correlation as a Function of Vapor Quality................................65 


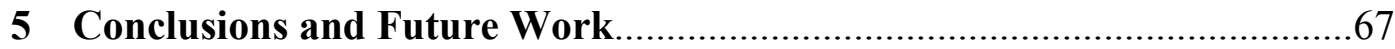

5.1 Conclusions with Regard to Flow Regime Maps..................................67

5.2 Conclusions with Regard to Heat Transfer Correlations...........................69

5.3 Future Work in Flow Regime Maps and Heat Transfer Correlations........70

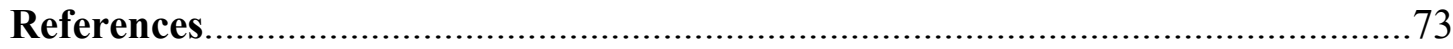




\section{List of Figures}

1.1 Schematic representation of flow regimes in shear-driven and gravity-driven flow condensers through a rectangular channel with condensation on the

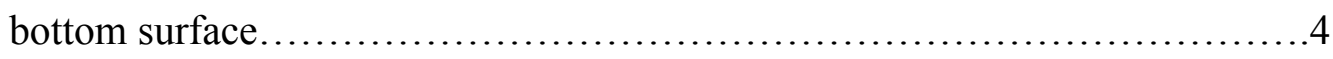

1.2 Flow regimes in horizontal flow condensation with transverse gravity in a

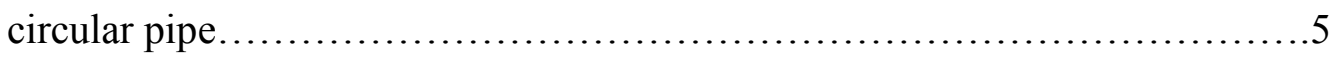

2.1 Schematic of internal condensing flow in a microgravity configuration through a rectangular channel................................................ 9

3.1 A schematic of the extents of the stratified/annular regime in the $\left\{R e_{i n}, \frac{\mathrm{Ja}}{\operatorname{Pr}_{1}}, \hat{x}\right\}$

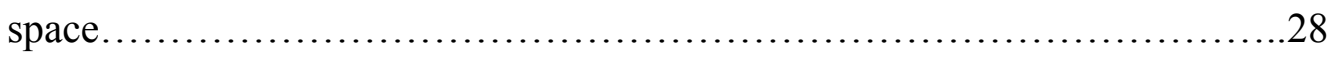

3.2 An equivalent map of the 3 dimensional flow regime in the Fig. 3.1 in the 2 dimensional $\left\{R e_{i n}, X\right\}$ domain as presented in various flow regime studies....30

3.3 Flow regime map for horizontal gas-liquid flow as proposed by Taitel and Dukler in the log-log scale. 34

3.4 Annular-misty, bubbly and intermittent (slug-plug) flow regimes in the $\left\{R e_{i n}, X\right\}$ domain as interpreted from the Taitel and Dukler map. .38 
3.5 Stratified/Annular, annular-misty, intermittent (slug-plug) flow regimes in the $\left\{R e_{i n}, X\right\}$ domain as interpreted from the Taitel and Dukler map

3.6 Schematic of modified Taitel and Dukler Map for consistence with

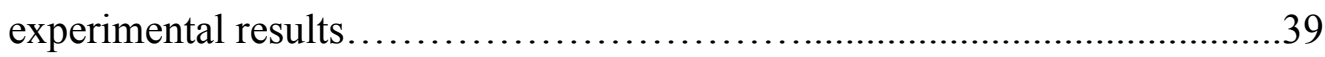

3.7 Transformed version of Fig. 3.6 in the $\left\{\mathrm{Re}_{\mathrm{in}}, \mathrm{X}\right\}$ domain.....................39

3.8 Flow regime map as proposed by Baker in the log-log scale ..................42

3.9 The different flow regimes shown in the $\left\{R e_{i n}, X\right\}$ domain as interpreted from the Baker map for the fluid R-134A..............................................................43

3.10 Close-up of the transformed Baker map in the $\left\{R e_{i n}, X\right\}$ domain to show the extents of the Stratified/Annular flow regime

3.11 Schematic of the modified Baker Flow Regime Map for consistence with experimental results. 46

3.12 Transformed version of Fig. 3.11 in the $\left\{R e_{i n}, X\right\}$ domain..............................46

3.13 Agreement between the variation of vapor quality with non-dimensional distance obtained from the quasi-1D simulation tool and the solution of Eq. 3.17 49 
3.14 Different flow regimes shown in the $\left\{\frac{J a}{P r_{1}}, R e_{i n}, \hat{x}\right\}$ space obtained by a conversion of data from the modified Baker map in Fig. 3.12 for the fluid R134A

4.1 Variation of the local heat transfer coefficient as a function of vapor quality obtained from Kim and Mudawar correlation..............................................58

4.2 Variation of the local heat transfer coefficient as a function of vapor quality obtained from Koyama et al. correlation.....................................................60

4.3 Variation of the local heat transfer coefficient as a function of vapor quality obtained from Cavallini and Zecchin correlation.......................................61

4.4 Comparison between the correlation developed by this study (Eq. 4.18) and some popular engineering heat transfer correlations.......................66 


\section{Acknowledgements}

I am extremely grateful to my advisor, Dr. Amitabh Narain for having supported me in every way for the two years that I spent under his tutelage. Without his ideas and encouragement this work would never have taken shape.

I am also thankful to Ranjeeth Naik for always being there to help me with the implementation of ideas into code. Without his expertise with the research tools used in this study, completion of this research would have been immensely harder.

I would like to thank Siddharth Ravikumar for his collaboration in this research and for running the simulations for many cases whose results were required for this research.

I am grateful to Dr. Sunil Mehendale for providing help with this research during the initial stages when there were lot more questions than answers. His contribution in compiling available engineering correlations in literature helped initiate this research.

I would also like to thank Dr. VC Rao Komaravolu for agreeing to be a part of my thesis defense committee and encouraging me to complete the work on time.

I would finally like to thank all my friends and family, who have been a constant source of moral and emotional support. I could not imagine my life without them. 


\section{Abstract}

Several modern-day cooling applications require the incorporation of mini/microchannel shear-driven flow condensers. There are several design challenges that need to be overcome in order to meet those requirements.

The difficulty in developing effective design tools for shear-driven flow condensers is exacerbated due to the lack of a bridge between the physics-based modelling of condensing flows and the current, popular approach based on semi-empirical heat transfer correlations. One of the primary contributors of this disconnect is a lack of understanding caused by the fact that typical heat transfer correlations eliminate the dependence of the heat transfer coefficient on the method of cooling employed on the condenser surface when it may very well not be the case. This is in direct contrast to direct physics-based modeling approaches where the thermal boundary conditions have a direct and huge impact on the heat transfer coefficient values. Typical heat transfer correlations instead introduce vapor quality as one of the variables on which the value of the heat transfer coefficient depends. This study shows how, under certain conditions, a heat transfer correlation from direct physics-based modeling can be equivalent to typical engineering heat transfer correlations without making the same apriori assumptions.

Another huge factor that raises doubts on the validity of the heat-transfer correlations is the opacity associated with the application of flow regime maps for internal 
condensing flows. It is well known that flow regimes influence heat transfer rates strongly. However, several heat transfer correlations ignore flow regimes entirely and present a single heat transfer correlation for all flow regimes. This is believed to be inaccurate since one would expect significant differences in the heat transfer correlations for different flow regimes. Several other studies present a heat transfer correlation for a particular flow regime - however, they ignore the method by which extents of the flow regime is established.

This thesis provides a definitive answer (in the context of stratified/annular flows) to: (i) whether a heat transfer correlation can always be independent of the thermal boundary condition and represented as a function of vapor quality, and (ii) whether a heat transfer correlation can be independently obtained for a flow regime without knowing the flow regime boundary (even if the flow regime boundary is represented through a separate and independent correlation). To obtain the results required to arrive at an answer to these questions, this study uses two numerical simulation tools the approximate but highly efficient Quasi-1D simulation tool and the exact but more expensive 2D Steady Simulation tool. Using these tools and the approximate values of flow regime transitions, a deeper understanding of the current state of knowledge in flow regime maps and heat transfer correlations in shear-driven internal condensing flows is obtained. The ideas presented here can be extended for other flow regimes of shear-driven flows as well. Analogous correlations can also be obtained for internal condensers in the gravity-driven and mixed-driven configuration. 


\section{Background and Introduction}

\subsection{Need for Research in Flow Condensers}

There is a critical need for thermal management systems with reduced mass and size that are capable of handling ever-increasing high heat loads associated with fine temperature control and heat-flux removal [1]. This need is particularly evidenced in requirements of the high-power electronics industry - which needs both condensers and boilers of reduced mass, size and high heat load capability [2, 3]. The inability of standard cooling techniques to dissipate the heat generated by high-power electronics is causing limitations in the development of smaller electronic systems with larger power densities [3]. For high performance electronic cooling requirements, and performance requirements of other high performance thermal management systems (in ground-based or space/aviation based applications), the authors in $[3,4]$ discuss a variety of promising thermal management technologies that are emerging as possible solutions to the problem of high heat-flux $(\sim 100$ $\mathrm{W} / \mathrm{cm}^{2}$ ) removal in these applications. After a comparison of various technologies, they $[3,4]$ present phase-change flows, i.e. flow condensation and flow boiling (with water as the operating fluid) as the technology with the greatest potential for providing an effective solution. 
Thermal management systems that use this phenomenon of phase change by evaporation and condensation of a fluid in a closed loop require flow-condensers and flow-boilers as indispensable elements within this closed loop. Reliable design of flow-condensers and flow-boilers, and their effective integration in closed loop thermal management systems require dependable flow prediction capabilities and strategies for effective flow control.

The research presented in this thesis aims to address some of the issues associated with the predictive capabilities for flow-condensation and hopes to help in the better design of modern flow condensers (such as the ones described in [2]).

\subsection{Design-related Issues associated with Internal Condensing Flows}

Flow-condensers can be operated in various configurations. Depending on the configuration in which a flow-condenser is operated, the primary forces driving the flow may vary. Traditional macro-scale internal flow condensers operated in a vertical or inclined orientation relative to Earth's gravitational vector give rise to what are known as gravity-driven or gravity-dominated flows. Macro-scale flowcondensers operated in a horizontal configuration may or may not be gravity dominated. For example, they are gravity dominated for slow inlet flow rates (associated with stratified/annular flows) and are shear/pressure driven for faster 
inlet flow rates (associated with annular flows). However, modern micro-scale flowcondensers operated in any orientation give rise to shear/pressure driven flows [5]. Gravity-driven flows are generally more stable and results related to gravity driven flow condensers are well known and published (see [5, 6]). Gravity-driven flows primarily occur in the stratified/annular flow regime for both channel and tube flows (see Fig. 1.1). In shear/pressure driven flow-condensers, the force of gravity is essentially absent and hence the flow of the liquid condensate is driven primarily by shear force between the liquid film and the vapor each flowing at different velocities. This absence of gravity in shear/pressure driven flow condensers readily causes flow regimes (see Figs. 1.1 and 1.2) inside the condenser that are undesirable because they are thermally inefficient. These inefficient flow regimes are commonly categorized as bubbly flow, slug flow and plug flow (see Figs. 1.1 and 1.2) in the gas-liquid flows experimentally studied in literature ([7-12]). Analogous flow regimes are observed in internal condensing flows. It should be noted that there are several subtle variations in the terminologies used for the different flow regimes.

The flow morphology plays a critical role in the determination of heat and mass transfer in internal condensing flows. One of the objectives of this study is to use the understanding from the gas-liquid flows studied in literature to predict the location of the flow regimes observed in internal condensing flows. The goal of a high performance condenser is to lengthen the stratified/annular flow regime and to operate entirely in this regime. Hence it is important to know the locations where 
the transition occurs from one regime to another. Several flow regimes are available in literature ([7-12]) that provide quick and rough estimates to identify the conditions when the transition between different flow regimes occurs. The fundamental structure of some of these flow regime maps is described in detail in section 3 .

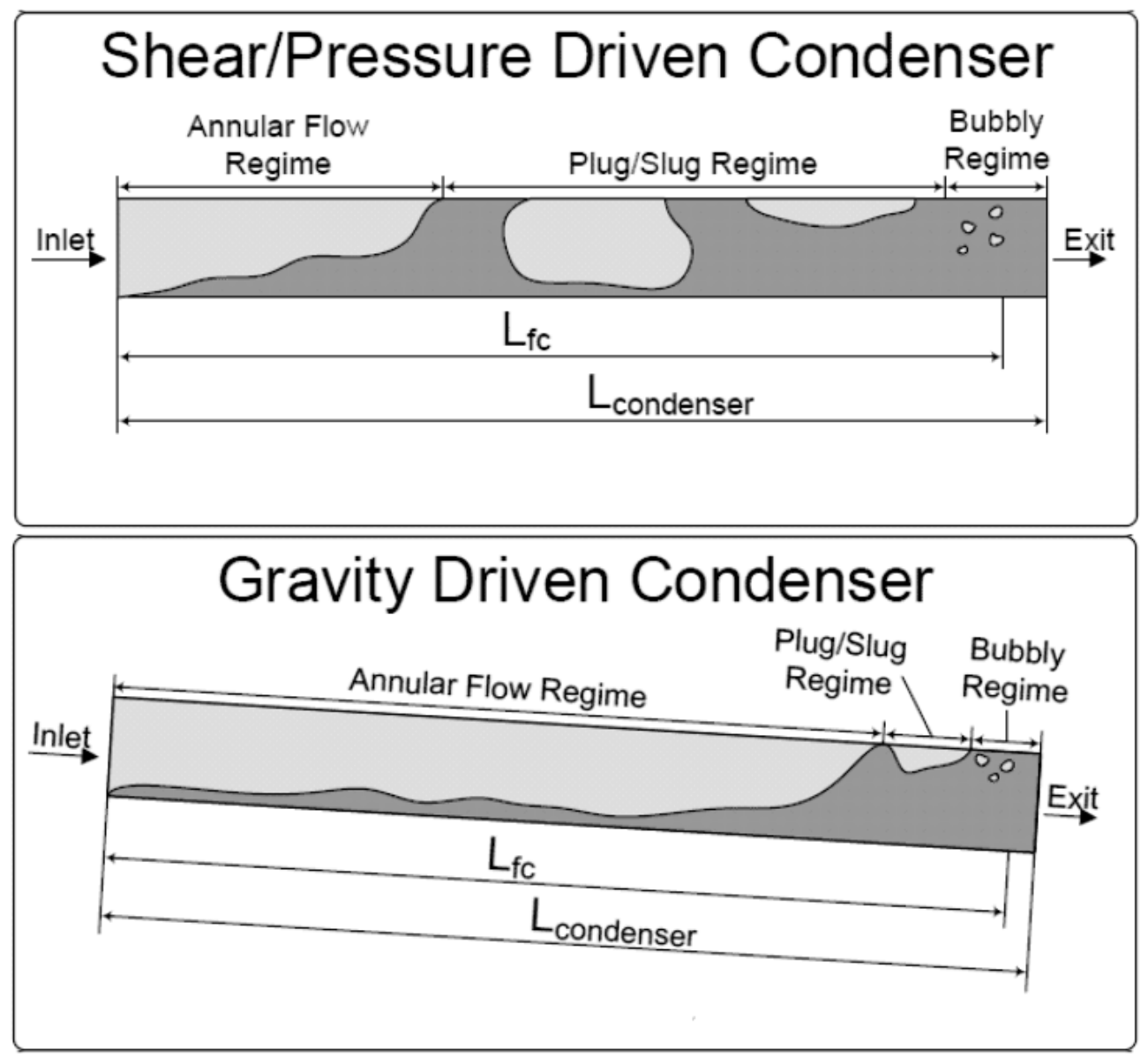

Figure 1.1: Schematic representation of flow regimes in shear-driven and gravitydriven flow condensers through a rectangular channel with condensation on the bottom surface 


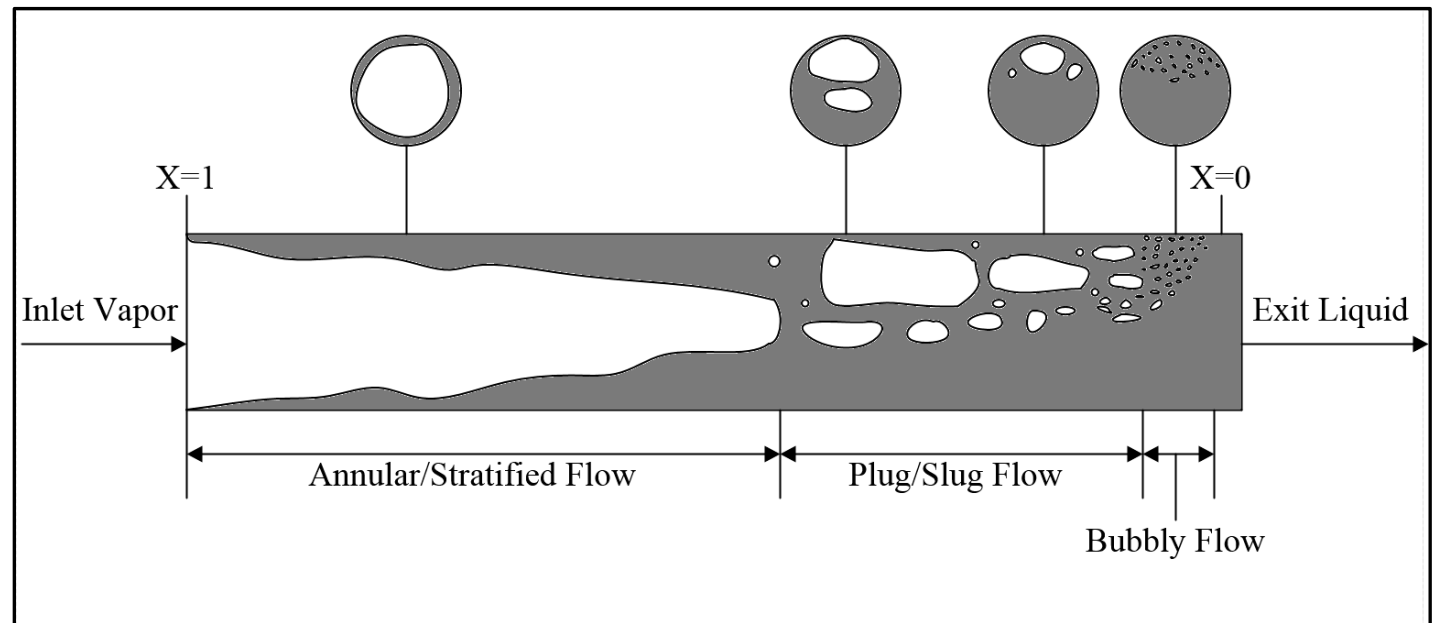

Figure 1.2: Flow regimes in horizontal flow condensation with transverse gravity in a circular pipe ([13])

Once the conditions favorable for realizing stratified/annular flow are identified, an estimate for the heat-transfer coefficient is required. This information is necessary for designing condensers for the operating heat loads. There are several semiempirical correlations available in literature ([14-18]) that present equations to calculate/estimate the heat-transfer coefficient. Most of these studies on the heattransfer coefficient focus on heat-transfer in the stratified/annular regime since that is the primary heat-load carrying flow regime, particularly among those present in the traditional operations of flow condensers. It may be noted here that the research group of Dr. Amitabh Narain at Michigan Technological University has proposed 
innovative condenser operations that ensure stratified/annular flow regimes along the entire length of the condenser [2].

The second objective of this study is to examine the validity of these semi-empirical heat-transfer correlations. An assessment of the structure of some of these heattransfer correlations is conducted in section 3 . The results from the studies available in literature ([14-18]) are compared with results that are obtained in this study using a combination of an approximate Quasi-1D condensing flow simulation tool ([5, 19]) and an exact scientific 2D steady simulation code ([19-23]). The mathematical models and the two simulation tools used in this study are described in section 2 . 


\section{Predictive Capabilities for Flow-Condensers}

\subsection{Computational Tools Used for this Study}

As mentioned in section 1, the goal of this study is to examine the principles underlying flow regime identification maps/correlations and the corresponding heat transfer correlations available in literature, assess their validity, and compare their results to those obtained from numerical simulations. Most of the studies available on flow regime maps and heat transfer coefficients in literature have been obtained by developing semi-empirical correlations. Although some of these correlations are more general than others, all of them have restrictions in either fluid properties or operating conditions due to restrictions in fluids and/or operating conditions in the experiments that supplement the correlations and due to the empirical nature of the studies conducted. Professor Amitabh Narain's research group at Michigan Technological University has pioneered the development and use of numerical simulation techniques for the study of flow condensers. These are quite accurate (for small wave, stratified/annular regimes) and have been validated by experimental studies undertaken by the same group. Several simulation tools have been developed over the years $([5,19-27])$. The details of each of these computational tools will not 
be presented in this thesis. However, the governing equations solved to obtain results for condensing flows and the important characteristics of the simulation tools used for this study - the quasi $1 \mathrm{D}$ simulation tool $([5,19])$ and the steady $2 \mathrm{D}$ scientific simulation tool ([19-23]) will be described here.

\subsection{Theoretical Model for Internal Condensing Flows}

The problem being solved is of condensing flow through a horizontal rectangular channel (Fig. 2.1) with or without transverse gravity under different operating conditions.

The fluids used to obtain results in this study have been limited, by choice, to water, FC-72, R113 and R134A. Pure vapor of the refrigerant at its saturation temperature, $T_{\text {sat }}\left(P_{\text {in }}\right)$, corresponding to an inlet pressure of $\left(P_{i n}\right)$ flows into the duct with a velocity of $U_{i n}$. The bottom plate in Fig. 2.1 is the condensing surface and is held at a temperature lower than the saturation temperature of the inlet vapor. This is done either by coolant circulation under the condensing surface or by the use of ThermoElectric Coolers (TECs). The upper plate is a non-condensing surface and is held at a temperature that is slightly higher than the saturation temperature of the inlet fluid. This is to offset any cooling of the vapor phase associated with heat losses to the surrounding wall and to approximate a constant temperature of the vapor phase. 


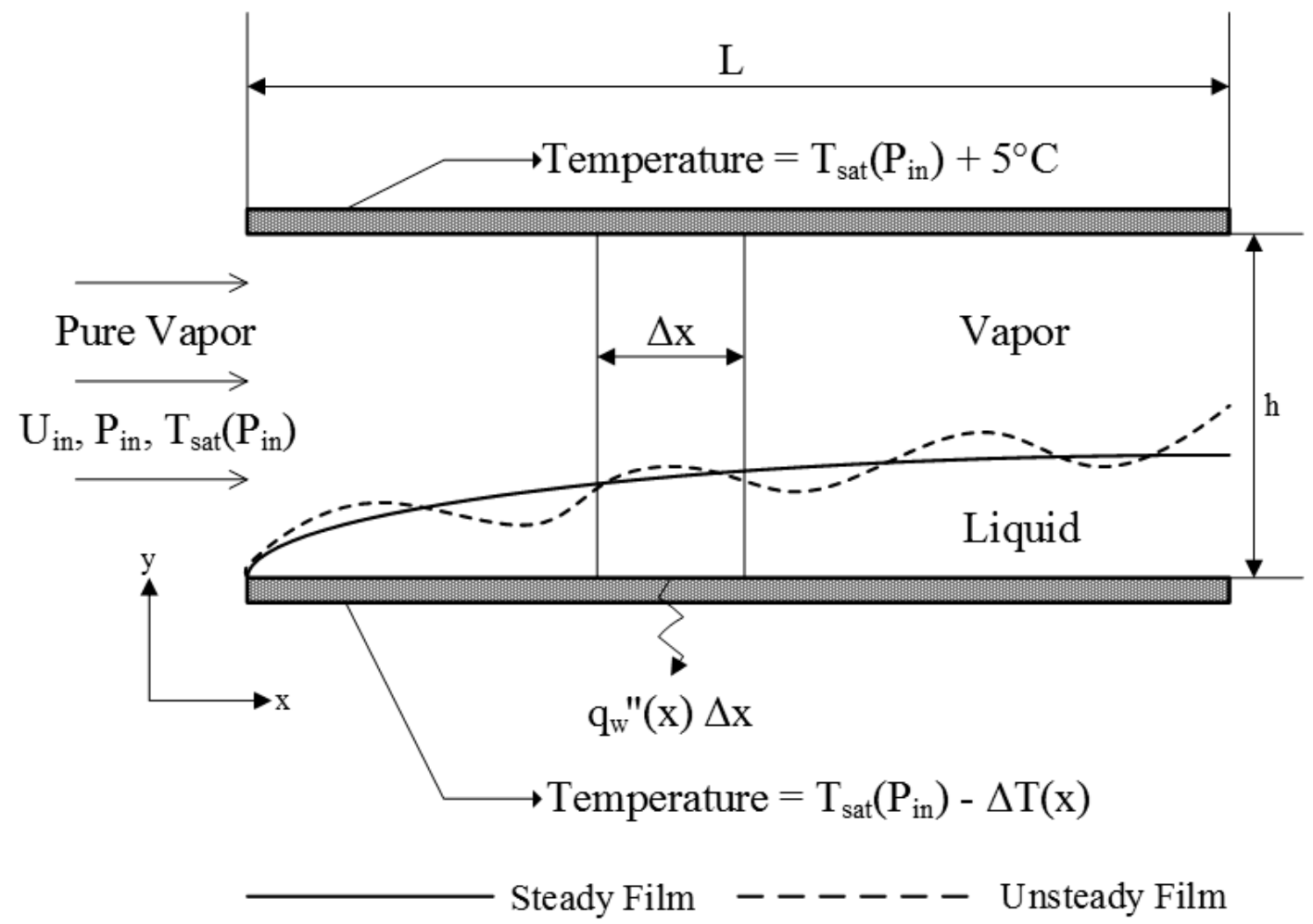

Figure 2.1: Schematic of Internal condensing flow in a microgravity configuration through a rectangular channel

Due to the lower temperature of the condensing surface, the inlet vapor condenses on the bottom plate and forms a liquid film as shown in Fig. 2.1. Both the quasi-1D and 2D simulations used for this study are steady simulations and can only capture the smooth steady film shown in Fig. 2.1. As the inlet flow rate is increased in the stratified/annular flow regime, the liquid-vapor interface is caused to become wavy as also shown in Fig. 2.1. This study does not intend to analyze the wave effects associated with the liquid-vapor interface but instead focuses on using the steady simulation tools to use the flow regime maps in the true experimental domain (see 
section 3) and also to obtain theoretical correlations for heat transfer in the annular/stratified flow regime of interest (see section 4).

These steady simulation tools cannot capture the transient effects associated with the wavy liquid-vapor interface and loss of annularity. To conduct an analysis of the unsteady wave dynamics of the liquid-vapor interface, an exact unsteady 2D simulation tool has been pioneered by Professor Amitabh Narain's research group at Michigan Technological University ([20, 22-23]).

The steady simulation tools are used in this study to predict the location of the steady liquid-vapor interface, the values of flow variables of the liquid and vapor phase, and the heat transfer rates at the condensing surface. Assuming an all vapor flow at the inlet $(X=1)$ where the onset of condensation occurs, the vapor quality of the flow is also computed at different $x$ locations from the inlet $(x=0)$ of the channel. This parameter, vapor quality, is important to be computed since almost all the studies related to flow regime maps and heat transfer correlations available in literature describe the parameters of interest as functions (either implicit or explicit) of the vapor quality (or equivalently the liquid quality, $1-X$ ). These are described in detail in section 3.

The variables in the liquid and vapor phases of the condensing flows described here are denoted with the subscript $I$, where $I=1$ for the liquid phase and $I=2$ for the vapor phase. The fluid properties used as inputs to this model (for each phase) are the fluid density $\rho$, dynamic viscosity $\mu$, specific heat $C_{p}$ and thermal conductivity 
$k$. These fluid properties are modeled as constants for each phase $(I=1$ or $I=2)$ over the entire length of the condenser being considered.

The inlet velocity is assumed to be parallel to the axis of the condenser ( $\mathrm{x}$-axis in Fig. 2.1) and is denoted by $U_{i n}$. The inlet pressure and inlet temperature are denoted by $P_{\text {in }}$ and $T_{\text {sat }}\left(P_{\text {in }}\right)$ as shown in Fig. 2.1. The inlet temperature is close to the saturation temperature and is effectively treated as being equal to the saturation temperature. The temperature fields in the interior of each phase are denoted by $T_{I}$, pressure fields by $P_{I}$, and the velocity fields by $\mathbb{V}_{I}=U_{I} \boldsymbol{i}+V_{I} \boldsymbol{j}$ (where $\boldsymbol{i}$ and $\boldsymbol{j}$ are are unit vectors along the $\mathrm{x}$-axis and $\mathrm{y}$-axis shown in Fig. 2.1). The film thickness is denoted by $\Delta$ and the local interfacial mass flux by $\dot{m}$. The temperature of the bottom condensing plate shown in Fig. 2.1 is denoted by $T_{w}(x)=T_{\text {sat }}\left(P_{\text {in }}\right)-$ $\Delta T(x)$ where $\Delta T(x)$ is the local difference between the saturation temperature of the incoming vapor and local wall temperature. The heat of vaporization is denoted by $h_{f g}$. The length and height of the channel are denoted by $L$ and $h$ respectively (see Fig. 2.1).

It should be noted that all the variables mentioned in the earlier paragraph are variables with physical dimensions (in SI units unless mentioned otherwise). In order to simplify the generalization of equations for any unit system, we use the non-dimensional form of the governing equations by introducing the following nondimensional variables [19]: 
$\left\{x, y, \Delta, U_{I}, \dot{m}\right\} \equiv\left\{L \hat{x}, L \hat{y}, L \delta, U_{i n} u_{I}, \rho_{1} U_{i n} \widehat{m}\right\}$

$\left\{V_{I}, T_{I}, P_{I}\right\} \equiv\left\{U_{i n} v_{I}, \overline{\Delta T} \theta_{I}, P_{i n}+\pi_{I} \rho_{1} U_{i n}{ }^{2}\right\}$

where $\hat{x}, \hat{y}, \delta, u_{I}, \widehat{m}, v_{I}, \theta_{I}$ and $\pi_{I}$ are the non-dimensional forms of the variables $x, y, \Delta, U_{I}, \dot{m}, V_{I}, T_{I}, P_{I}$.

\subsection{Two Dimensional Governing Equations for Condensing Flow}

In order to solve for the flow variables within each phase (i.e. liquid and vapor) of the condensing flow, the governing equations used are the steady, incompressible, two-dimensional forms of the well-known differential equations for mass, momentum and energy balance. These equations are written in their corresponding non-dimensional forms as:

$$
\begin{aligned}
& \frac{\partial u_{I}}{\partial \hat{x}}+\frac{\partial v_{I}}{\partial \hat{y}}=0 \\
& u_{I} \frac{\partial u_{I}}{\partial \hat{x}}+v_{I} \frac{\partial u_{I}}{\partial \hat{y}}=-\left(\frac{\partial \pi_{I}}{\partial \hat{x}}\right)+F r_{x}^{-1}+R_{I}^{-1}\left(\frac{\partial^{2} u_{I}}{\partial \hat{x}^{2}}+\frac{\partial^{2} u_{I}}{\partial \hat{y}^{2}}\right) \\
& u_{I} \frac{\partial v_{I}}{\partial \hat{x}}+v_{I} \frac{\partial v_{I}}{\partial \hat{y}}=-\left(\frac{\partial \pi_{I}}{\partial \hat{y}}\right)+F r_{y}^{-1}+R e_{I}^{-1}\left(\frac{\partial^{2} v_{I}}{\partial \hat{x}^{2}}+\frac{\partial^{2} v_{I}}{\partial \hat{y}^{2}}\right)
\end{aligned}
$$


$u_{I} \frac{\partial \theta_{I}}{\partial \hat{x}}+v_{I} \frac{\partial \theta_{I}}{\partial \hat{y}}=\operatorname{Re}_{I}^{-1} \operatorname{Pr}_{I}^{-1}\left(\frac{\partial^{2} \theta_{I}}{\partial \hat{x}^{2}}+\frac{\partial^{2} \theta_{I}}{\partial \hat{y}^{2}}\right)$

where $R e_{I} \equiv \rho_{I} U_{i n} h / \mu_{I}, \operatorname{Pr}_{I} \equiv \mu_{I} C_{p I} / k_{I}, F r_{x}^{-1} \equiv g_{x} h / U_{\text {in }}^{2}$ and $F r_{y}^{-1} \equiv g_{y} h / U_{\text {in }}^{2}$.

Here Eq. (2.2) is the incompressible mass balance equation, Eq. (2.3) is the $\mathrm{x}$ and $\mathrm{y}$ components of the momentum balance equation and Eq. (2.4) is the energy balance equation. It should be noted that the terms related to the time-varying nature of the variables have been dropped from the equations. The full steady interior governing equations contain the body force parameters related to the accelerations due to gravity along the $\mathrm{x}$ and $\mathrm{y}$ axes of the condenser (as shown in Fig. 2.1). Hence both the simulation tools used in this study can be used to predict the results for macroscale inclined channel flows too (gravity-driven flows). However, since this study is only interested in modern flow condensers in a microgravity application, i.e. in shear/pressure driven internal condensing flows, the gravity parameters of $\mathrm{Fr}_{x}^{-1}$ and $\mathrm{Fr}_{y}^{-1}$ will always be equal to zero for all results described in this study.

The solution of these equations in each incompressible phase (for $I=1$ and $I=$ 2) give the values of the variables within each phase. However, an additional set of equations is needed to determine the extents of each phase - i.e. by finding the location of the liquid-vapor interface that forms the boundary between the two phases. The steady interface is described using an explicit equation:

$\phi \equiv y-\Delta(x)=0$ 
The accurate model for interface conditions associated with condensing flows are well known and are given in [21]. The interface conditions using the superscript " $\mathrm{i}$ " for the flow variables at the interface location are rewritten below.

- The continuity equation for the tangential components of velocities is given by:

$u_{2}^{i}=u_{2}^{i}-\delta_{\hat{x}}\left(v_{2}^{i}-v_{1}^{i}\right)$

where $\delta_{\hat{x}} \equiv \partial \delta / \partial \hat{x}$

- The momentum balance in the normal component at the interface is given by:

$\pi_{1}^{i}=\frac{\rho_{2}}{\rho_{1}} \pi_{2}^{i}-W e^{-1}\left(\frac{\delta_{\mathfrak{x} \chi}}{\left[1+\delta_{\grave{\chi}}^{2}\right]^{1.5}}\right)+\dot{m}^{2}\left(\frac{\rho_{1}}{\rho_{2}}-1\right)$

where $W e \equiv \rho_{1} U_{\text {in }}^{2} h / \sigma$, and surface tension $\sigma=\sigma(\mathcal{T})$ where $\mathcal{T}$ is the temperature at the interface.

- The momentum balance in the tangential component at the interface is given by:

$\left.\frac{\partial u_{1}}{\partial \hat{y}}\right|^{i}=\left.\frac{\mu_{2}}{\mu_{1}} \frac{\partial u_{2}}{\partial \hat{y}}\right|^{i}+[t]$

The definition of term $[t]$ is taken from Eq. (A.9) of [21]

- Constraints on the interfacial velocities of the liquid and vapor phase are imposed by the non-zero interfacial mass fluxes $\widehat{m}_{L K}$ and $\widehat{m}_{V K}$ as defined in Eq. (A5) of [21]. These kinematic constraints are given by: 


$$
\begin{aligned}
& \widehat{m}_{L K}=\left(u_{1}^{i} \frac{\partial \delta}{\partial \hat{x}}-v_{1}^{i}\right) / \sqrt{1+\left(\frac{\partial \delta}{\partial \hat{x}}\right)^{2}} \\
& \widehat{m}_{V K}=\left(\frac{\rho_{2}}{\rho_{1}} u_{2}^{i} \frac{\partial \delta}{\partial \hat{x}}-v_{2}^{i}\right) / \sqrt{1+\left(\frac{\partial \delta}{\partial \hat{x}}\right)^{2}}
\end{aligned}
$$

- The interfacial mass flux $\widehat{m}_{\text {energy }}$, as defined in Eq. (A.6) of [21] represents the constraint on the temperature imposed by the net transfer of energy through the interface. This is given by:

$$
\widehat{m}_{\text {energy }} \equiv \frac{J a}{\operatorname{Re}_{1} P r_{1}}\left[\left.\frac{\partial \theta_{1}}{\partial n}\right|^{i}-\left.\left(\frac{k_{2}}{k_{1}}\right) \frac{\partial \theta_{2}}{\partial n}\right|^{i}\right]
$$

Here $J a \equiv C_{p 1} \Delta T / h_{f g}^{0}$ and $h_{f g}^{0} \equiv h_{f g}\left(T_{s a t}\left(P_{\text {in }}\right)\right) \cong h_{f g}\left(T_{\text {sat }}\left(P_{2}^{i}\right)\right)$

- Mass balance at the interface requires that the overall mass flux across the interface be the same value irrespective of the physical process used to obtain it (see [19]). This is expressed in non-dimensional forms as:

$$
\widehat{m} \equiv \widehat{m}_{L K}=\widehat{m}_{V K}=\widehat{m}_{\text {energy }}
$$

- The thermodynamic restriction on the interfacial temperatures as in Eq. (A.8) of [21] is given by:

$$
\theta_{1}^{i} \cong \theta_{2}^{i}=\frac{T_{\text {sat }}\left(P_{2}^{i}\right)}{\Delta T} \equiv \theta_{\text {sat }}\left(\pi_{2}^{i}\right)
$$

It should be noted that the changes in pressures of the vapor phase occurring within the condenser are large enough to affect vapor motion but are negligible compared 
to the inlet pressure of the vapor. Therefore the changes in saturation temperature of the vapor associated with the change in vapor pressures within the condenser can be ignored. Thus:

$T_{\text {sat }}\left(P_{2}^{i}\right)=T_{\text {sat }}\left(P_{i n}\right)$, and correspondingly,

$\theta_{\text {sat }}\left(\pi_{2}^{i}\right)=\theta_{\text {sat }}\left(\pi_{\text {in }}\right)$

These system of equations described by Eqs. (2.2) - (2.13) cannot be solved without the knowledge of boundary conditions and inlet conditions that complete the problem mathematically. These conditions that need to be satisfied (and required to solve the system of equations numerically) at each boundary are described below.

The temperature of the top plate of the condenser is assumed to be constant with a value that is slightly larger than the saturation temperature of the vapor (which is assumed to be constant). This enables the assumption of a nearly constant temperature (equal to the saturation temperature) of the vapor phase throughout the condenser. The effects of superheating the top surface of the condenser (in the range of $5-10^{\circ} \mathrm{C}$ ) are not important and can be neglected. The no-slip wall boundary condition is also imposed on the top wall $\left(u_{2}\left(\hat{x}, \frac{h}{L}\right)=v_{2}\left(\hat{x}, \frac{h}{L}\right)=0\right)$.

For the bottom plate of the condenser, besides the no-slip wall boundary condition $\left(u_{1}(\hat{x}, 0)=v_{1}(\hat{x}, 0)=0\right)$, the thermal boundary condition describing the temperature of the condensing surface is also prescribed. In non-dimensional form it is prescribed as: 
$\theta_{1}(\hat{x}, 0)=\theta_{w}(x) \equiv T_{w}(x) / \Delta T$

where $T_{w}(x)=T_{\text {sat }}\left(P_{\text {in }}\right)-\Delta T \equiv \overline{T_{w}} \boldsymbol{\varphi}(x)$ and $\boldsymbol{\varphi}(x)$ is a non-dimensional function of $x$. For instance, if the local temperature difference is constant along the length of the condenser, i.e. if $T_{w}(x)$ is independent of $x$, then $\boldsymbol{\varphi}(x)$ would be a constant function with $\varphi(x)=1$ (the identity function).

For the inlet boundary at $x=0$, we have a uniform velocity profile, $U_{\text {in }}$ and therefore:

$u_{2}(0, \hat{y})=1$, and

$\left.\frac{\partial v_{2}}{\partial \hat{x}}\right|_{\hat{x}=0}=0$

It should be noted that the inlet pressure is not prescribed along the entire inlet boundary but is only given at the corner where the inlet boundary and the top wall intersect $[5,19]$. The value of the inlet pressure $P_{\text {in }}$ appears indirectly through thermodynamic properties such as $h_{f g}\left(P_{2}^{i}\right) \cong h_{f g}\left(P_{\text {in }}\right)$ and $T_{\text {sat }}\left(P_{2}^{i}\right) \cong T_{\text {sat }}\left(P_{\text {in }}\right)$ (see [19]). 


\subsection{Two-Dimensional Steady Condensing Flow Solver}

The governing equations describing the problem to be solved described in Eqs. (2.2)

- (2.15) in section 2.3 has been developed (see [21]) by the research group at Michigan Technological University led by Dr. Amitabh Narain. The interior equations of each phase (liquid and vapor) are solved using COMSOL Multiphysics, a commercial Finite Element Analysis (FEA) package. The interface between the two phases is solved in MATLAB (from the MathWorks, Inc.) with information being exchanged between COMSOL and MATLAB. The tool uses an initial guess of the interface location and iterates between COMSOL and MATLAB to solve the interior equations and also find the true converged location of the interface iteratively. This algorithm and the complexities associated with obtaining the solution are not described here since that is beyond the scope of this study. Details on the computational methodology can be found in [19-23].

This study uses the results of this exact 2D steady computational tool to supplement results obtained from the Quasi 1D steady computational tool. Although the 1D tool is fairly accurate and largely agrees with the results from the 2D code, it is developed through an approximate theory (described in section 2). Thus to be completely sure of the results and conclusions of this study, the results from the $2 \mathrm{D}$ code were used to replace/improve the results from the 1D code for certain key 
points (primarily the extents) of the parameter space in the stratified/annular regime being studied.

\subsection{D Approximation for the Complete 2D Governing Equations}

The Quasi 1D technique for solutions to condensing flow equations used in this study is unlike other 1D approximations to condensing flow such as the ones in [2830]. These popular techniques [28-30] techniques use average variables for the flow and incorporate empirical models (such as friction factor models for the interface, pressure gradient models, certain turbulence models, etc.) that are assumed to be true (see [5]) for the solution technique. The 1D tool used in this study is called the quasi 1D steady condensing flow tool because it uses the exact analytical functions for laminar/laminar, annular/stratified condensing flow by making a single approximation on the vapor profile $u_{2}(\hat{x}, \hat{y})$ with regard to its cross sectional (i.e. $\hat{y}$ ) variation [19]. Integral forms of vapor phase momentum equation and the vapor phase mass balance equation alone are used. This minimizes the impact of this $\hat{y}$ variation assumption on $u_{2}(\hat{x}, \hat{y})$ on the one dimensional variables of interest that naturally arise in the analytical solution of the liquid domain equations.

The 2D non-dimensional momentum and energy equations in differential form (see Eqs. 2.3 - 2.4) for the liquid phase condensate $(I=1)$ get simplified for the $1 \mathrm{D}$ case 
under the assumption of the nearly flat, thin film, boundary layer approximation $\left(\frac{\partial}{\partial \hat{x}} \ll \frac{\partial}{\partial \hat{y}}\right.$ and $\left.v_{I} \ll u_{I}\right)$, negligible inertial momentum terms and negligible convection energy term $[5,19]$. Based on these assumptions, the 2D steady momentum (the $x$ and $y$ components in Eq. 2.3) and the energy equations (Eq. 2.4) simplify to:

$0 \cong u_{1} \frac{\partial u_{1}}{\partial \hat{x}}+v_{1} \frac{\partial v_{1}}{\partial \hat{x}} \cong \frac{1}{R e_{1}} \frac{\partial^{2} u_{1}}{\partial \hat{x}^{2}}-\frac{\partial \pi_{1}}{\partial \hat{x}}+F r_{x}^{-1}$

$0 \cong \frac{\partial \pi_{1}}{\partial \hat{y}}-F r_{y}^{-1}$

$0 \cong \frac{1}{R e_{I} P r_{I}}\left(\frac{\partial^{2} \theta_{I}}{\partial \hat{y}^{2}}\right)$

Here the two equations in Eq. 2.16 are the simplified momentum balance equation and Eq. 2.17 is the simplified energy balance equation.

Furthermore, the formulation used in the quasi-1D simulation tool assumes that the cross-sectional pressure variation in the vapor phase to be uniform [5]. This leads to:

$P_{2}=P_{2}(x)=P_{0}+\rho_{2} U^{2} \pi_{2}(\hat{x})$

where $\pi_{2}(\hat{x}) \equiv \pi$ and $\pi(0) \equiv 0$.

The formulation also assumes no impact of the superheated top surface of the vapor and negligible interfacial slope approximation [19]. This also simplifies the interfacial conditions and results in the modified interfacial conditions given below: 


$$
\begin{aligned}
& u_{2}^{i}=u_{1}^{i}=u_{f}(\hat{x}) \\
& \pi_{1}^{i}=\frac{\rho_{2}}{\rho_{1}} \pi_{2}^{i}=\frac{\rho_{2}}{\rho_{1}} \pi(\hat{x}) \\
& \left.\frac{\partial u_{1}}{\partial \hat{y}}\right|^{i}=\left.\frac{\mu_{2}}{\mu_{1}} \frac{\partial u_{2}}{\partial \hat{y}}\right|^{i} \\
& \hat{m}_{\text {energy }} \cong \frac{J a}{R e_{1} P r_{1}}\left(\left.\frac{\partial \theta_{1}}{\partial \hat{y}}\right|^{i}\right)
\end{aligned}
$$

These equations (Eqs. $2.16-2.22$ ) are solved to obtain a solution for the onedimensional variables of interest like velocity, pressure, temperature and film thickness. The analytical solutions and the setting up of the problem for solution are not presented here. References $[5,19]$ can be referred to for details on the algorithm.

\subsection{Quasi-1D Steady Condensing Flow Simulation Tool}

The equations mentioned in section 2.5 (Eqs. 2.16 - 2.22) can be solved for multiple pipe and channel geometries, and for a variety of fluids and flow conditions. This quasi-1D tool, though based on an approximate technique, is sufficiently accurate and has been validated by comparisons with results from the 2D steady tool and also with results from experimental runs (see [5]). This tool is extremely versatile over a range of fluids and operating conditions, and offers solutions to the condensing flow 
problem with a low computational cost without compromising on the accuracy of the results.

The quasi-1D steady tool is extensively used in this study - this is due to the solver speed and sufficient accuracy of the results obtained. Numerous shear-driven condensing flow cases were run on this tool to obtain quick results which were used in conjunction with a popular flow regime map to generate our own understanding of what a general flow regime map (see section 3) would look like and also to obtain the restrictions on our heat transfer correlation (see section 4) for the stratified/annular flow regime. The boundaries of this regime cannot, at present, be obtained solely through computational analysis.

The quasi-1D solver uses Mathematica (Wolfram Research, Inc) which is a symbolic manipulation software to perform algebra which simplifies the formulation. This manipulation results in a set of four first order ordinary differential equations which are solved on MATLAB. The details of this formulation manipulation and the computational algorithm are not mentioned here since they are not the primary focus of this study. They are described in detail in [5, 19]. 


\section{Flow Regimes in Shear-Driven Internal Condensing}

\section{Flow}

\subsection{Introduction to Flow Morphology and the Importance of Flow Regimes in Shear-Driven Internal Condensing Flows}

In internal condensing flows, the liquid and vapor are in motion simultaneously inside the channel or pipe in consideration. This causes more complicated physics in two-phase flows than that of single phase flows. Along with the typical inertial, viscous and pressure forces present in single-phase flow, interfacial tension forces, wetting characteristics of the condensate liquid on the walls of the condenser, and momentum exchange between the liquid and vapor phases are important in twophase flows and need to be considered [13]. The arrangement of the two-phases in internal condensing flow can be quite complex - particularly in shear-driven internal condensing flows. This arrangement of the phases is typically referred to as the flow morphology or flow regime. The flow morphology plays an important role in determining the performance of the condenser and in general depends of the condenser geometry, fluid properties, flow operating conditions and the configuration of the flow-condenser in operation. 
In studies of the flow morphology in internal condensing flows, the presence or absence of a particular flow morphology is popularly monitored as a function of the dryness fraction or vapor quality for all flow conditions kept constant. This quality is sometimes also referred to as the thermodynamic quality. Henceforth referred to simply as quality, $X$ or $X(x)$, this parameter is defined, at any location, as the ratio of vapor flow rate, $\dot{M}_{v a p}(x)$ to the total flow rate in the condenser, $\dot{M}(x)$.

$\dot{M}(x)=\dot{M}_{v a p}(x)+\dot{M}_{l i q}(x)$

$X(x)=\frac{\dot{M}_{v a p}(x)}{\dot{M}(x)}$

Since this study focuses solely on shear-driven internal condensing flows, the flow morphology associated with gravity-driven flows will not be discussed here. One may refer to [13] for details on flow morphology and heat transfer correlations for the easier to model gravity driven condensers in a vertical orientation.

For shear-driven internal condensing flows, the flow regimes typically observed are shown in Figs 1.1 and 1.2. The description of flow regimes as a function of quality is given in [13]. This description is essentially an extension of the mapping approach based on flow regimes observed in gas-liquid two phase flows without phase change (i.e. air-water type flows). The flow regimes observed in actual condensing flows is described in [13] and well as in the results of the experiments of Dr. Narain's experimental research group at Michigan Technological University [2, 31-34]. In their results, obtained for experiments where $R e_{i n}$ values vary within the 
range of 4000 to 17000 and $J a / P r_{1}$ values vary between 0.01 to 0.026 , stratified flow (sometimes called annular flow) is observed at high values of quality, $X$. As the value of $X$ drops, large pockets of vapor start to form giving rise to plug/slug flow. It should be noted that plug and slug flow are not considered to be distinct flow regimes for the results in [2]. At even lower values of $X$, there is a lot more liquid with many smaller vapor bubbles resulting in what is termed as bubbly flow. This is observed even for the smaller $R e_{\text {in }}$ values in the range of $4000-17000$.

It should be noted here that the term annular flow is typically reserved for pipe flows where, at high flow rates, the liquid surrounds the pipe wall of the condenser allowing vapor flow through the interior - giving rise to an annulus. Hence the term annular flow. In rectangular channel condensers, the condensing surface is only the bottom plate and therefore the liquid condensate doesn't form an annulus like in pipe flow. However, the stratified flow regime prevails even at larger flow rates in channel flow-condensers and hence for channels we will refer to this stratified regime as stratified/annular.

Several studies have proposed flow regimes maps based on the consideration of different parameters [7-12]. These maps are sufficiently easy to interpret and give a good estimate of the qualities at which a certain flow will exist - assuming all other defining flow parameters are known and constant. This assumption is based on the understanding obtained from empirical research done with gas-liquid flows (with no phase change). 
At even larger flow rates, the liquid-vapor interface becomes wavy and is termed as wavy stratified/annular flow. This study does not make a fine distinction between smooth stratified/annular and wavy stratified/annular since the difference in the heat transfer rates between the two regimes can be empirically handled without having to undertake an unsteady simulation of condensing flows. This empirical treatment of the correlation for the heat transfer coefficient of wavy stratified/annular flows is described in section 4. Hence as long as the interface remains continuous and stratified, this study calls it stratified/annular (whether smooth or wavy).

Since flow regimes play a huge impact on the heat transfer rates in internal condensing flows, it is of critical importance to interpret and modify the results of flow regimes in gas-liquid flows by properly considering the additional physics associated with two phase flows with heat and mass transfer across the interface (i.e. condensing and boiling flows). Details on the how studies on heat transfer correlations are associated with the studies on flow regime maps for internal condensing flows are given in section 3.2. 


\subsection{Association of Heat Transfer Correlations with Studies on Flow Regimes in Internal Condensing Flows}

The studies relating to flow regime maps and heat transfer correlations associated with internal condensing flows sometimes requires the users of these maps and correlations to place unwarranted faith when using the results obtained from these studies. This study aims to question some of the assumptions made by a typical study that relates to flow regime maps and heat transfer correlations and to provide answers that improve the current state of knowledge in this field.

In order to understand the basic premise of most of the studies, consider a fixed set of $\left\{\frac{\rho_{2}}{\rho_{1}}, \frac{\mu_{2}}{\mu_{1}}\right\}$ values and a fixed function describing the spatial variation of the wall temperature, $\boldsymbol{\varphi}(\hat{x})$. Now consider variations in the $\left\{R e_{i n}, \frac{J a}{P r_{1}}, \hat{x}\right\}$ space that correspond to variations in the inlet flow rate, temperature difference and the downstream distance along the length of the condenser. If one were to find the extents of the stratified/annular regime boundary within this space, it would be defined by the length of the annular regime obtained for each point in the $\left\{R e_{i n}, \frac{J a}{P r_{1}}\right\}$ plane. A schematic of such a section of the $\left\{R e_{i n}, \frac{J a}{P r_{1}}, \hat{x}\right\}$ space is shown in Fig. 3.1 - where the surface shaded in grey and bounded by points $\left\{1^{\prime}, 2^{\prime}, 3^{\prime}, 4^{\prime}\right\}$ is $\hat{x}_{A}$ value for

each point in the set of $\left\{R e_{i n}, \frac{J a}{P r_{1}}\right\}$ points considered - which is shown by the 
bottom surface bounded by points $\{1,2,3,4\} . \hat{x}_{A}$ corresponds to the value of the nondimensional length of the annular/stratified regime for a particular point in the $\left\{R e_{i n}, \frac{\overline{J a}}{P r_{1}}, \frac{\rho_{2}}{\rho_{1}}, \frac{\mu_{2}}{\mu_{1}}, \llbracket \boldsymbol{\varphi}(\hat{x}) \rrbracket\right\}$ space. $\llbracket \boldsymbol{\varphi}(\hat{x}) \rrbracket$ is the set of all possible non-dimensional functions that $\varphi(\hat{x})$ may assume.

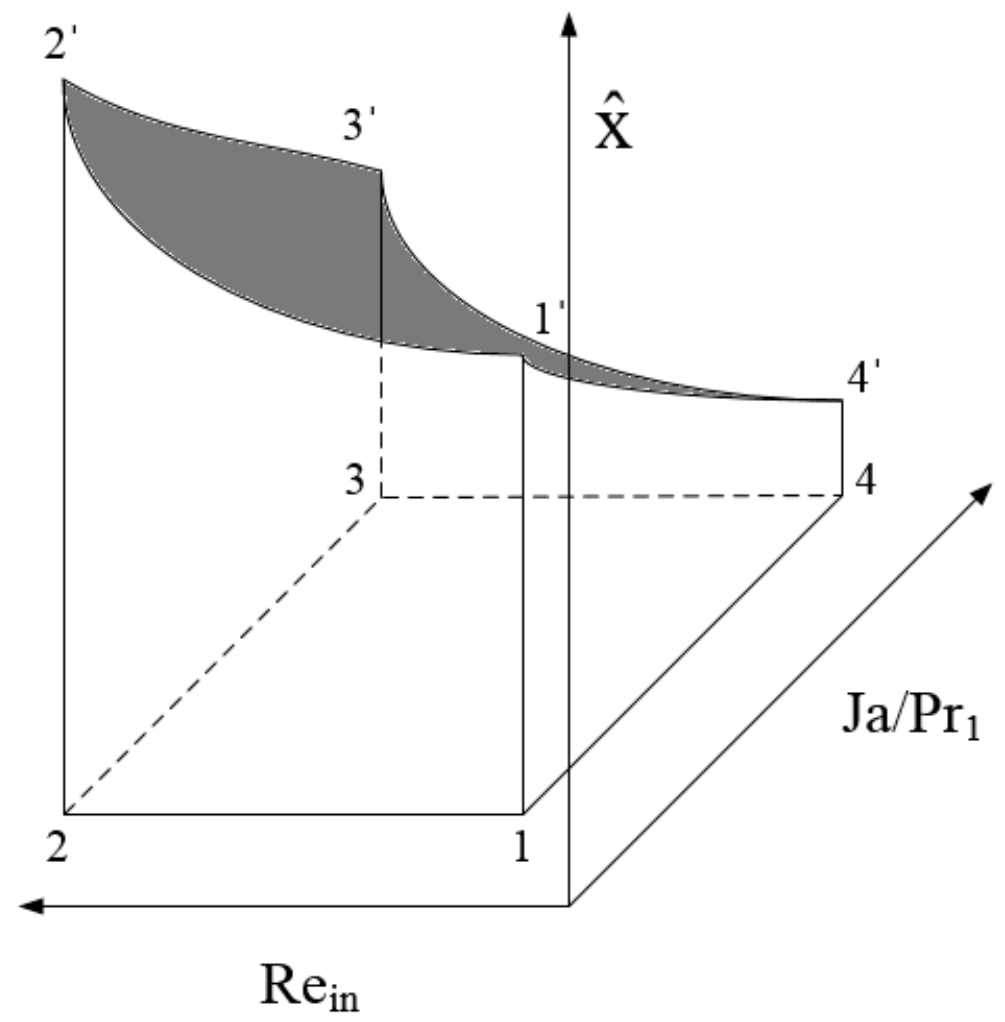

Figure 3.1: A schematic of the extents of the stratified/annular regime in the $\left\{R e_{i n}, \frac{J a}{P r_{1}}, \hat{x}\right\}$ space 
It can be seen in Fig. 3.1 that $\left.\hat{x}_{A}\right|_{2,}>\left.\hat{x}_{A}\right|_{1}$, because $\left.\mathrm{Re}_{\text {in }}\right|_{2,}>\left.\mathrm{Re}_{\text {in }}\right|_{1}$, while $\left.\frac{J a}{P r_{1}}\right|_{1},=\left.\frac{J a}{P r_{1}}\right|_{2}$. This is expected since the length of the annular regime increases with increase in the inlet vapor speed for a given value of temperature difference (assuming that the values of $\frac{\rho_{2}}{\rho_{1}}, \frac{\mu_{2}}{\mu_{1}}, \llbracket \boldsymbol{\varphi}(\hat{x}) \rrbracket$ are also the same). Similarly it can be seen that $\left.\hat{x}_{A}\right|_{1,}>\left.\hat{x}_{A}\right|_{4,}$ because while $\left.\operatorname{Re}_{\mathrm{in}}\right|_{1,}=\left.\operatorname{Re}_{\mathrm{in}}\right|_{4 \prime},\left.\quad \frac{J a}{P r_{1}}\right|_{1,}<\left.\frac{J a}{P r_{1}}\right|_{4,}$. This again makes sense since the vapor condenses with haste when the temperature difference is high giving rise to non-annular regimes sooner. Thus the length of the annular regime increases with decreasing value of the temperature difference given all other parameters are the same.

The central thesis of flow regime maps is that the annular/stratified regime bounded by surfaces whose vertices are labeled by $\left\{1,2,3,4,1^{\prime}, 2^{\prime}, 3^{\prime}, 4^{\prime}\right\}$ in Fig. 3.1 are such that they can be equivalently mapped to a $\left\{R e_{i n}, X\right\}$ space (or an equivalent 2 dimensional space defined by liquid and vapor qualities) that is typically used in flow regime maps. Such an equivalent space which is also a schematic of a typical flow regime map for the annular/stratified regime in the $\left\{R e_{i n}, X\right\}$ space is shown in Fig 3.2. 


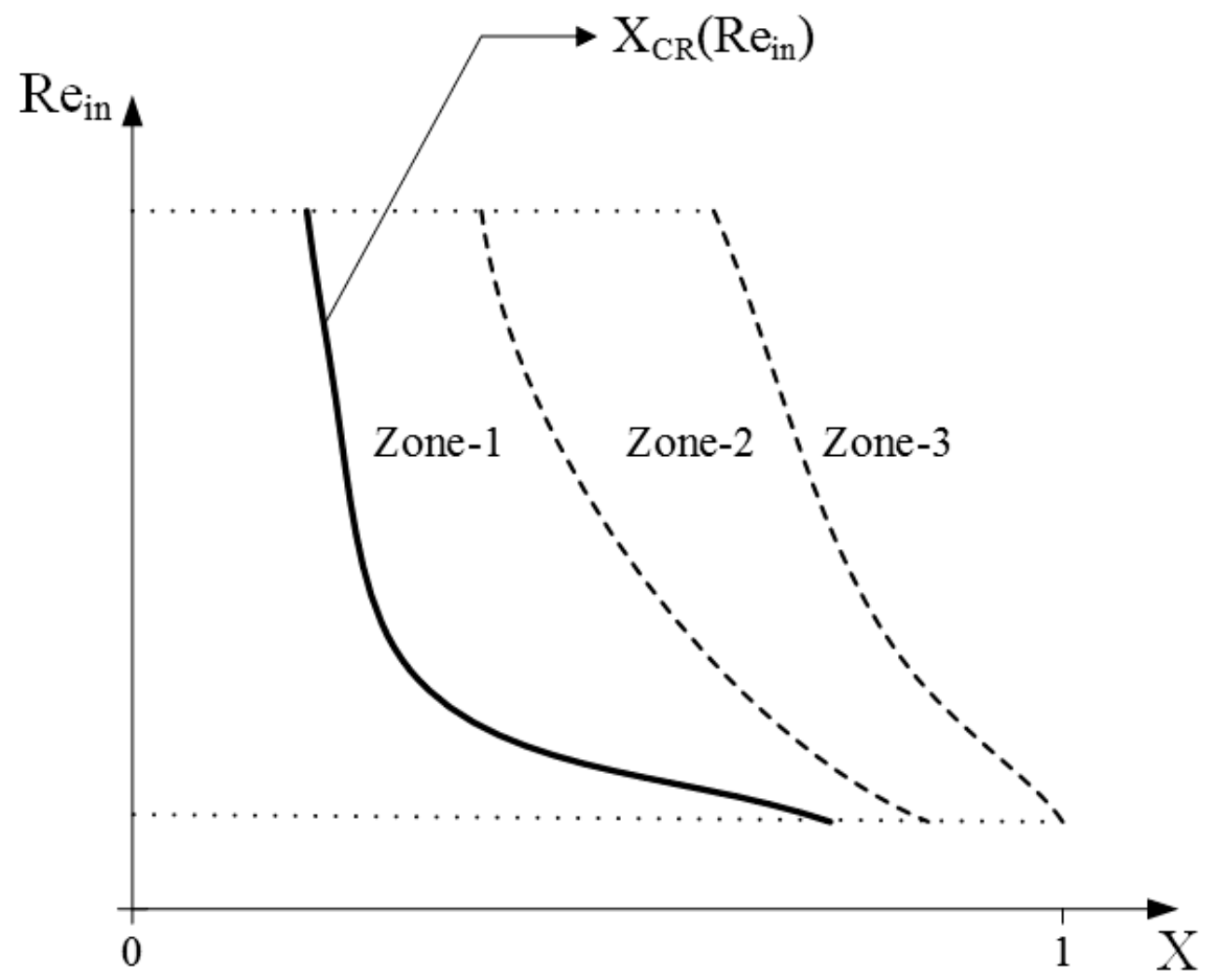

Figure 3.2: An equivalent map of the 3 dimensional flow regime in the Fig. 3.1 in the 2 dimensional $\left\{R e_{i n}, X\right\}$ domain as presented in various flow regime studies.

The annular/stratified zone in Fig. 3.2 may be defined by the region where $X \geq$ $X_{c r}\left(R e_{i n}\right)$. This region may be further bounded by curves (still undesignated) at the right, top and bottom of the bold curve. This is one of the common types of flow regime maps presented in literature. Another commonly observed type of two dimensional flow regime map relates the vapor mass flux within the pipe or channel to the liquid mass flux and presents flow regime transition curves in that space. The absence of any parameter related to the thermal boundary condition acting on the 
fluid is conspicuous and raises questions about the veracity of such maps (presented in literature) for all cooling conditions, no matter how vigorous (for example - a case where the condenser is cooled by high power thermo-electric coolers).

This fundamental idea employed by most flow regime maps in literature developed through the study of gas-liquid flows is carried forward by another idea put forth by several engineering heat transfer correlations. The local heat transfer coefficient in internal flow condensation is an important parameter to be considered for designing condensers that are required for the removal of a certain heat load determined by heat flux variations as defined by Eq. 3.3.

$q_{W}^{\prime \prime}(x) \equiv h_{x} \Delta T$

Here $q_{W}^{\prime \prime}(x)$ is the local heat flux at the wall (i.e. the bottom surface of Fig. 2.1), $\Delta T$ is the temperature difference as defined before, and $h_{x}$ is the local heat transfer coefficient.

The local heat transfer coefficient is a function of several parameters. This is stated in Eq. 3.4 for the definition of $h_{x}$ as a function of several physical parameters and in Eq. 3.5 by its non-dimensional form (using the Buckingham Pi Theorem) as a function of several non-dimensional parameters.

$$
\begin{aligned}
& h_{x} \equiv f\left(x, D_{h y d}, \dot{M}_{i n}, \sigma, T_{\text {sat }}\left(P_{i n}\right), \rho_{1}, \rho_{2}, \mu_{1}, \mu_{2}, k_{1}, k_{2}, C_{P 1}, C_{P 2}, h_{f g}\right) \\
& N u_{x} \equiv \frac{D_{h y d} h_{x}}{k_{1}} \equiv f\left(\hat{x}, R e_{i n}, \frac{J a}{P r_{1}}, \frac{\rho_{2}}{\rho_{1}}, \frac{\mu_{2}}{\mu_{1}}, \boldsymbol{\varphi}(\hat{x})\right)
\end{aligned}
$$


Note that Eq. 3.5 agrees with the parameter dependence suggested by the nondimensional form of the governing equations given in Eqs. 2.2 - 2.4. All of the empirical heat transfer correlations in literature present a correlation for the local heat transfer coefficient as a function of only a few of these non-dimensional parameters. A typical definition of the local heat transfer coefficient presented in literature avoids dependence on the boundary condition characterized by $J a$ and $\boldsymbol{\varphi}(\hat{x})\left(\right.$ or $\left.T_{\hat{x}}\right)$, and is given using a function such as the one in Eq. 3.6.

$\frac{D_{h y d} h_{x}}{k_{1}} \equiv f\left(X(\hat{x}), R e_{i n}, \frac{\rho_{2}}{\rho_{1}}, \frac{\mu_{2}}{\mu_{1}}\right)$

The primary goal of this study is to examine the validity of using maps such as the one indicated in Fig. 3.2 and the validity of using Eq. 3.6 for calculating the local heat transfer coefficient, $h_{x}$. If these approaches are valid, the next goal is to address the question about the relation between the two approaches - the physics based approach characterized by Fig. 3.1 and Eq. 3.5 and the other characterized by Fig. 3.2 and Eq. 3.6.

To fulfil the goals mentioned above, using the transition lines between different flow regimes published in the Taitel and Dukler map [7] and the Baker flow regime map [8], this study shows an ability to convert both maps to more easily readable maps in the $\left\{\operatorname{Re}_{i n}, X\right\}$ domain and compare the observed results with experimental results obtained for internal condensing flows in [2]. 
This study also uses the data from the Baker flow regime map [8] to generate a three dimensional flow transition map in the physics-based non-dimensional variables that correspond to the thermal boundary condition, $\Delta T(\hat{x})$, inlet flow velocity, $U_{\text {in }}$, and the distance downstream of the condenser, $x$ (see section 3.5). Once the physical domain map (correct extension of Fig. 3.1) for the annular/stratified flow regime is identified, different points on this domain are picked and solved for by using a combination of the quasi-1D tool and the 2D steady tool. This gives us all the required results to generate a heat transfer correlation for the stratified/annular flow regime and compare it to other semi-empirical heat-transfer correlations for this regime in literature (see section 4.2).

\subsection{Analysis of the Flow Regime Maps in Literature}

As mentioned in section 3.1, most flow regime maps in literature have arrived at their format of presentation of results for internal condensation through the extension of the presentation used for results obtained from the experimental study of gas-liquid flows. The physics of gas-liquid flows is considerably different from condensing flows - where there is the added complexity of heat and mass transfer across the interface which changes the physics of the flow. This is the reason that these maps seem counterintuitive for direct use in understanding condensing flow 
regimes since one would expect to see the effects of the thermal boundary conditions and physical distance just the way it is observed in experiments of flow condensation. In order to understand and verify the validity of such maps and to address the question of how the modeled flow regime maps (based on gas-liquid flows) relate to shear-driven internal condensing flows, the flow regime maps from $[7,8]$ have been studied here as representative examples. The inferences drawn by using data from these maps can be extended to flow regime maps for gravity-driven condensing flows, boiling flows, etc.

\subsubsection{The Taitel and Dukler flow regime map}

The Taitel and Dukler flow regime map published in 1976 [7] is widely regarded as being the more general one which has the most theoretical basis [13]. It is recreated based on data from the original article [7] in Fig. 3.3. 


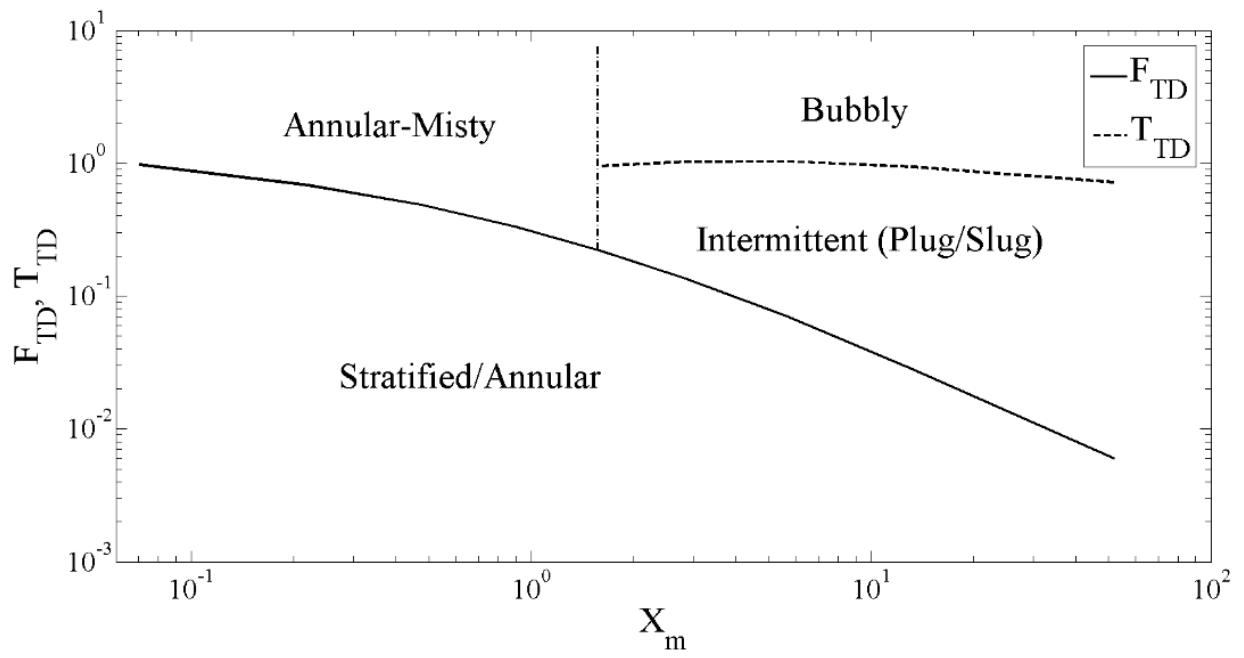

Figure 3.3: Flow regime map for horizontal gas-liquid flow as proposed by Taitel and Dukler [7] in the log-log scale

The horizontal co-ordinate on the Taitel and Dukler map is the Martinelli Parameter, $X_{m}$. The value of this parameter fixes the horizontal position of the map regardless of the flow regime. The Martinelli parameter, $X_{m}$ is defined as:

$X_{m}=\left[\frac{(d P / d x)_{l i q}}{(d P / d x)_{v a p}}\right]^{1 / 2}$

Here $(d P / d x)_{l i q}$ and $(d P / d x)_{v a p}$ are the frictional pressure gradients for the liquid and vapor phases flowing alone in the pipe. The values of these gradients can be computed by using the following equations which make use of the friction factor model:

$$
\left(\frac{d P}{d x}\right)_{l i q}=\frac{-2 f_{l} G^{2}(1-X)^{2}}{\rho_{1} D}
$$


$\left(\frac{d P}{d x}\right)_{v a p}=\frac{-2 f_{v} G^{2} X^{2}}{\rho_{2} D}$

$f_{l}=B R e_{1}^{-n}$

$f_{v}=B R e_{2}^{-n}$

$R e_{l}=\frac{G(1-X) D}{\mu_{1}}$

$R e_{v}=\frac{G X D}{\mu_{2}}$

In the Eqs. $3.4-3.9, G$ is the inlet vapor mass flux defined as $G \equiv 4 \dot{M} / \pi D_{\text {hyd }}^{2}$.

The friction factors $f_{l}$ and $f_{v}$ are determined experimentally and have been correlated for round pipes with different relations for laminar flow $\left(\operatorname{Re}_{1}\right.$ or $R e_{2}<$ $2000)$ and for turbulent flow $\left(R e_{1}\right.$ or $\left.R e_{2} \geq 2000\right)$. For laminar flow cases, $B=$ 16 and $n=1$. For turbulent flow cases, $B=0.0079$ and $n=0.25$. Most cases considered in this study are for turbulent flow, i.e. $R e_{1}$ or $R e_{2} \geq 2000$. The calculation of the Martinelli parameter using Eqs. 3.3 - 3.9 determines the location on the horizontal axis of the Taitel and Dukler flow regime map. The Eqs. 3.3 - 3.9 can be rewritten to show how the Martinelli parameter is a direct function of vapor quality for the specific range of turbulent Reynolds numbers mentioned. This relationship is given by Eq. 3.10 below:

$X_{m}^{8}=\frac{\mu_{l}}{\mu_{v}} \frac{(1-X)^{7}}{X^{7}}\left(\frac{\rho_{v}}{\rho_{l}}\right)^{4}$ 
The location on the vertical axis in Fig. 3.3 is determined by the value of a second non-dimensional parameter. This value also gives the location of the transition curve(s) between two flow regimes for a particular $X_{m}$ location on the horizontal axis. This dimensionless parameter could be one from a choice of three and is different based on the specific flow transition being considered.

In order to determine the flow regime transition from stratified/annular to annular dispersed (annular-misty) and intermittent (plug and slug) flow regimes, the nondimensional parameter used is $F_{T D}$. This parameter is defined by:

$$
F_{T D}=\left[\frac{\rho_{v} j_{v}^{2}}{\left(\rho_{l}-\rho_{v}\right) D g \cos \Omega}\right]^{0.5}
$$

For the map defining the flow transition from intermittent (plug and slug) to dispersed bubbly (bubbly regime), the dimensionless parameter to be used is $T_{T D}$. This is defined by:

$$
T_{T D}=\left[\frac{-(d P / d x)_{l}}{\left(\rho_{l}-\rho_{v}\right) D g \cos \Omega}\right]^{0.5}
$$

The third non-dimensional parameter is for the transition between the smooth and wavy stratified/annular regime. This regime is not of much interest to this study since both regimes taken together are of interest and termed as stratified/annular.

In Eq. 3.11 and 3.12, $g$ is the acceleration due to gravity and $\cos \Omega$ is the cosine of the angle of inclination of the condenser. Since this study only focuses on sheardriven internal condensing flows, the angle is taken to be $0^{\circ}$ (hence $\cos \Omega=1$ ). 
In Eq. 3.11, the term $j_{v}$ is referred to as superficial vapor flux and is defined as:

$$
j_{v}=\frac{\dot{m}}{A} \frac{X}{\rho_{v}}
$$

Using the definitions of the two axes in Fig 3.3 as given in Eqs. $3.3-3.13$, the curves denoting the transition between two flow regimes in these maps can be reconstructed to obtain approximate transformed curves in the $\left\{\operatorname{Re}_{i n}, X\right\}$ domain for a particular set of fluid properties and hydraulic diameter. For the sample transformed maps shown here, the fluid used was R-134A and a channel height of $0.002 \mathrm{~m}$.

Fig. 3.4 shows the annular-misty, bubbly and intermittent (plug-slug) flow regimes in a transformed map of Fig. 3.3 on the $\left\{R e_{i n}, X\right\}$ domain. This was calculated from interpreting the curves on the Taitel Dukler Map [7] (Fig. 3.3) using the Eqs. 3.3 3.13. The scale of Fig. 3.4 is such that the stratified/annular regime is barely visible. Fig. 3.5 shows the stratified/annular regime by changing the scale on Fig. 3.4. 


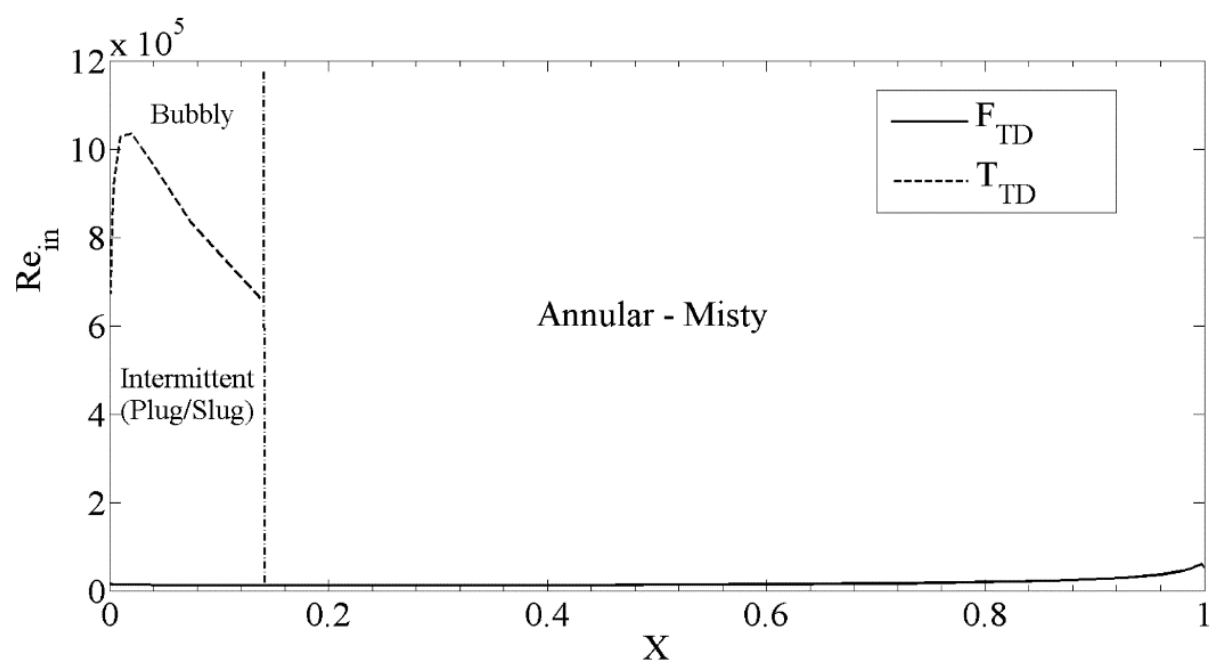

Figure 3.4: Annular-Misty, Bubbly and Intermittent (Plug-Slug) regimes in the $\left\{R e_{i n}, X\right\}$ domain as interpreted from the Taitel Dukler [7] map

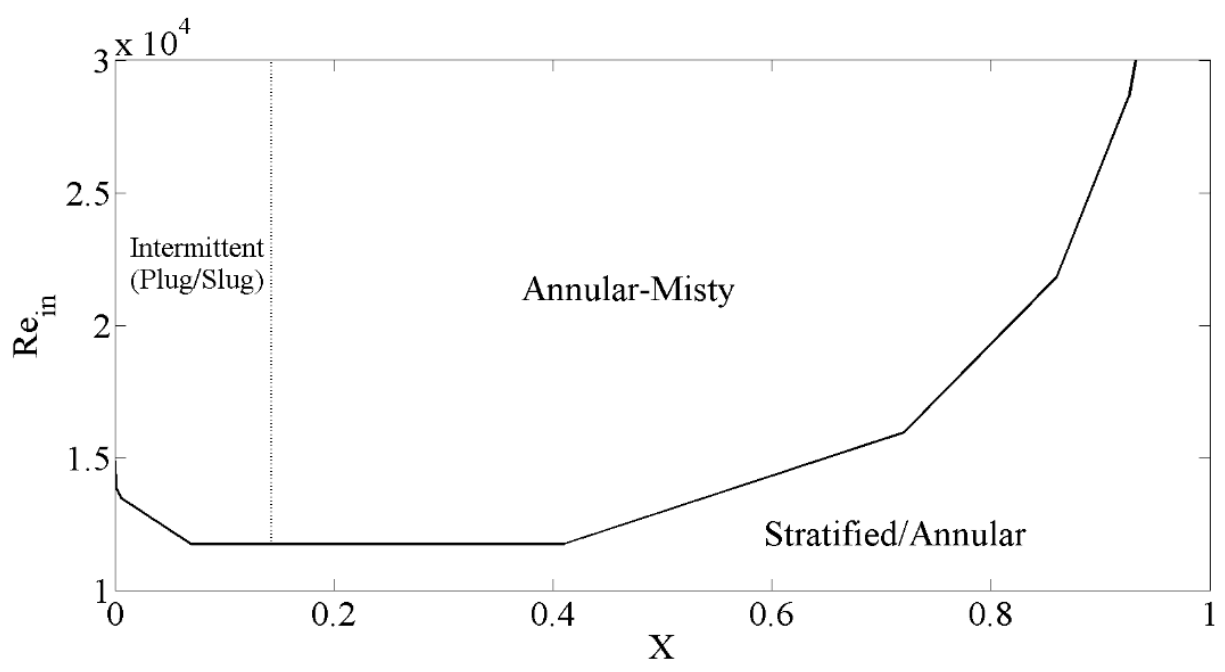

Figure 3.5: Stratified/Annular, Annular-Misty and Intermittent (Plug/Slug) regimes in the $\left\{R e_{i n}, X\right\}$ domain as interpreted from the Taitel Dukler map [7].

The transformed flow regime map observed in Figs. 3.4 and 3.5 do not seem to accurately describe the flow regimes in internal condensing flows for three reasons. The rationale for this distrust in the flow regimes observed in the transformed 
$\left\{\operatorname{Re}_{\mathrm{in}}, \mathrm{X}\right\}$ domain is described with the help of a Figs. 3.6 and 3.7. Fig 3.6 shows a modified schematic version of Fig. 3.3. Fig 3.7 then shows a transformed version of Fig. 3.6 in the $\left\{\operatorname{Re}_{\mathrm{in}}, \mathrm{X}\right\}$ domain.

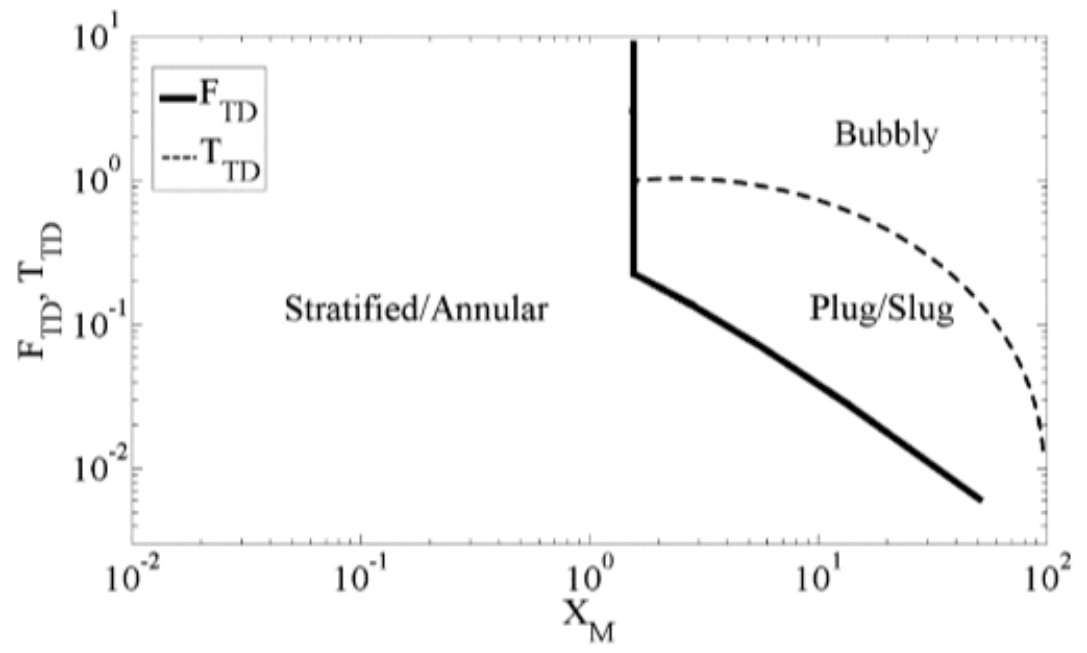

Figure 3.6: Schematic of modified Taitel and Dukler Map for consistence with experimental results of [2]

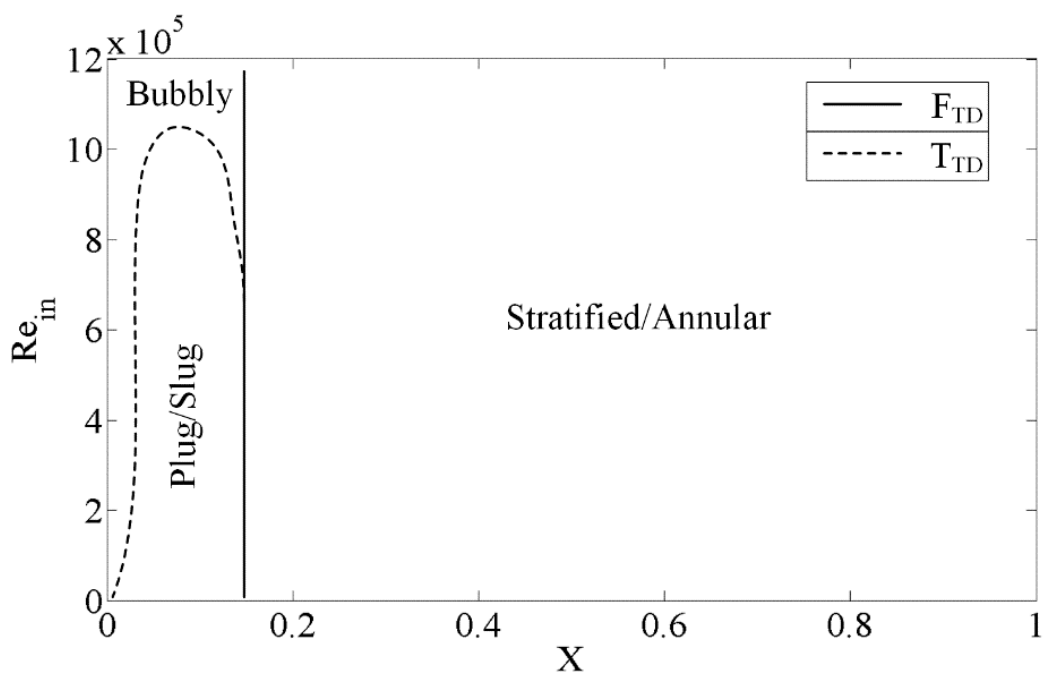

Figure 3.7: Transformed version of Fig. 3.6 in the $\left\{R e_{i n}, X\right\}$ domain 
The rationale for the modification is based on the observations in the experimental studies of internal condensing flows in [2]. The transition between the stratified/annular and the annular-misty as given by the Taitel Dukler map [7] (and in Fig 3.3) is not of great relevance to internal condensing flows. This is because, when there is an absence of heat and mass transfer across the interface, the liquid film is a lot more unstable causing annular-misty flow even at low inlet vapor speeds. The film is comparatively more stable for internal condensing flows. This hypothesis is corroborated by the absence of misty-annular regime in the experimental flow condensation results of [2]. However, since the results [2] are for lower values of inlet vapor speed $\left(R e_{i n}<20000\right)$, this study is unable to comment on the exact location of the transition between stratified/annular to annular-misty regime in the extension of Fig. 3.3 to internal condensing flows (Fig. 3.6).

It should also be noted that even for low inlet vapor speeds - as in the experiments performed in [2], when the quality drops very low, the flow regime transitions from plug/slug to bubbly. This is not seen in the transformed curves (Figs. 3.4, 3.5) of the original Taitel and Dukler flow regime map (Fig. 3.3). Hence one would expect a more accurate map to look similar to the maps shown in Figs 3.6 (for the original domain used by Taitel and Dukler [7]) and 3.7 (in the $\left\{\operatorname{Re}_{\mathrm{in}}, \mathrm{X}\right\}$ domain). 
Another reason that this study believes that Fig. 3.3 needs modification is because of the range of vapor qualities for which the stratified/annular regime is observed in Fig. 3.5 - even for very low inlet vapor speeds. When the inlet vapor speed drops below a certain value, the transition from annular to non-annular regimes is going to occur very quickly since there is no shear-force acting on the liquid film to keep it stable and stratified. This concern has also been addressed in the Fig. 3.7.

Thus the results observed from the Taitel and Dukler map [7] (see Fig. 3.3) seem qualitatively acceptable by making minor modifications. It is unfair to expect the Taitel and Dukler map [7] to be precisely accurate since (i) it is based on results obtained from the experimental investigations of gas-liquid flows (with no interfacial heat or mass transfer) and (ii) their range of inlet vapor speeds is so large that it seems probable that few cases if any involved experiments with low inlet vapor speeds where the primary inconstancies with the results of [2] are observed.

A similar study of the Baker flow regime map [8] is presented below in section 3.3.2.

\subsubsection{Baker Flow Regime Map}

Another popular flow regime map that is commonly used is the Baker flow regime map published in 1954 [8]. While this map is considered to be less general than the Taitel and Dukler flow regime map [7], it has benefits in that it is particularly useful 
for understanding the sequence of flow regimes observed in shear-driven internal condensing flows [13].

The axes used to describe the map and the different flow regimes are also different with this map. The horizontal axis of the Baker map is a dimensional property given by $G(1-X) \psi$ and the vertical axis is also a dimensional property $G X / \lambda$. Both the parameters have units of $\mathrm{ML}^{-2} \mathrm{~T}^{-2}$. Here $\psi$ and $\lambda$ are non-dimensional scaling parameters that account for variations in the fluid properties. $\psi$ and $\lambda$ are defined as:

$$
\begin{aligned}
& \psi=\left(\frac{\sigma_{w}}{\sigma}\right)\left[\left(\frac{\mu_{l}}{\mu_{w}}\right)\left(\frac{\rho_{w}}{\rho_{l}}\right)^{2}\right]^{1 / 3} \\
& \lambda=\left[\left(\frac{\rho_{v}}{\rho_{a}}\right)\left(\frac{\rho_{l}}{\rho_{w}}\right)\right]^{1 / 2}
\end{aligned}
$$

where the subscripts of $w$ and $a$ denote the properties for water and air respectively at room temperature and atmospheric pressure. $\sigma$ here denotes the value of surface tension (at the inlet pressure of 1 atm and at the saturation temperature corresponding to the inlet pressure) in units of force/unit length. A reconstruction of the Baker flow regime map is presented here in Fig. 3.8. 


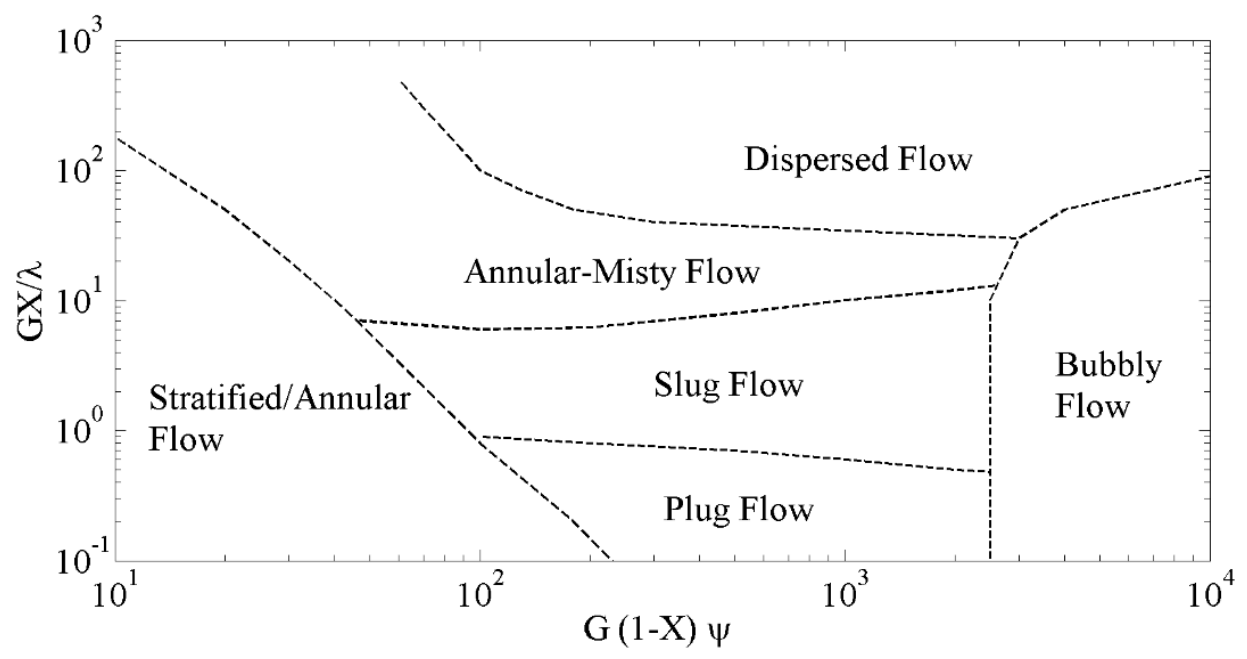

Figure 3.8: Flow regime map as proposed by Baker [8] in the log-log scale

The vertical axis in the Baker flow regime map is a measure of the vapor quality, $X$ of the two-phase flow while the horizontal axis is a measure of the liquid quality, $(1-X)$. Based on the values of the two quantities denoted by the axes of the Baker flow map, the transition between different flow regimes is depicted a curves on the map (as shown in Fig. 3.8). To determine the exact vapor quality at which a particular flow will transition to different flow regimes, the Baker map shown in Fig. 3.8. can also be transformed to a map with the Reynolds number of the inlet vapor $\left(R e_{i n}\right)$ on the vertical axis and vapor quality $(X)$ on the horizontal axis. This transformed plot is shown in Fig. 3.9 and Fig. 3.10. 


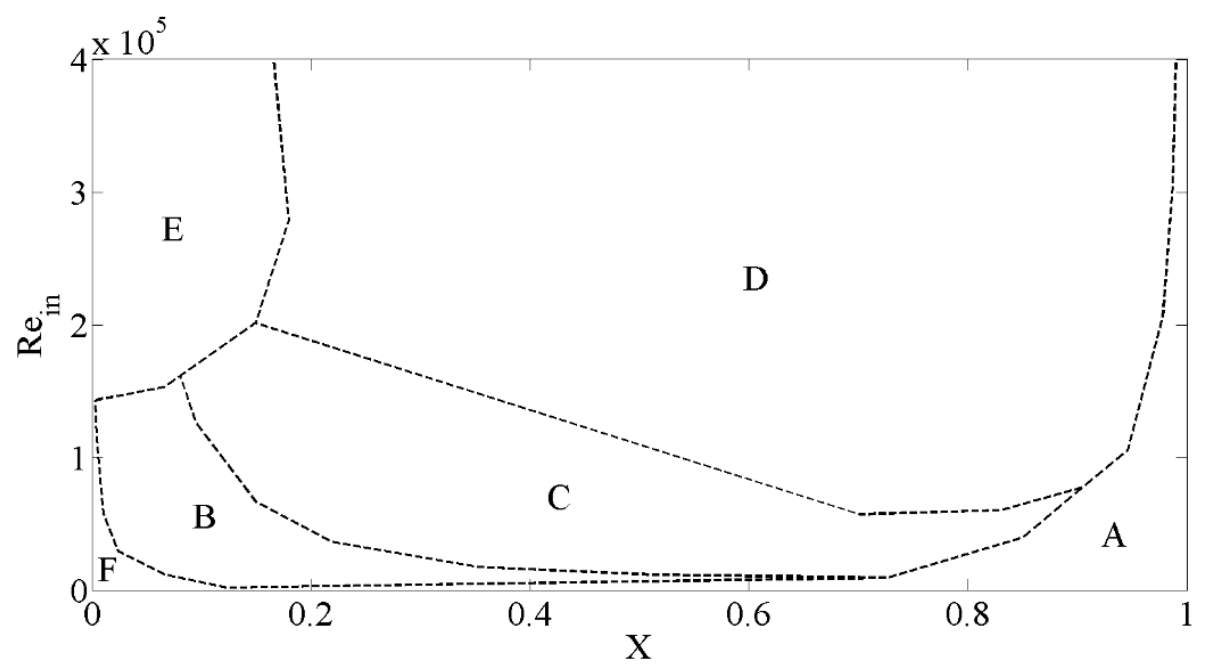

Figure 3.9: The different flow regimes shown in the $\left\{R e_{i n}, X\right\}$ domain as interpreted from the Baker map for the fluid R-134A. The zones are labeled as: A Stratified/Annular, B - Slug, C - Misty Annular, D - Dispersed, E - Bubbly and F Plug flow regimes.

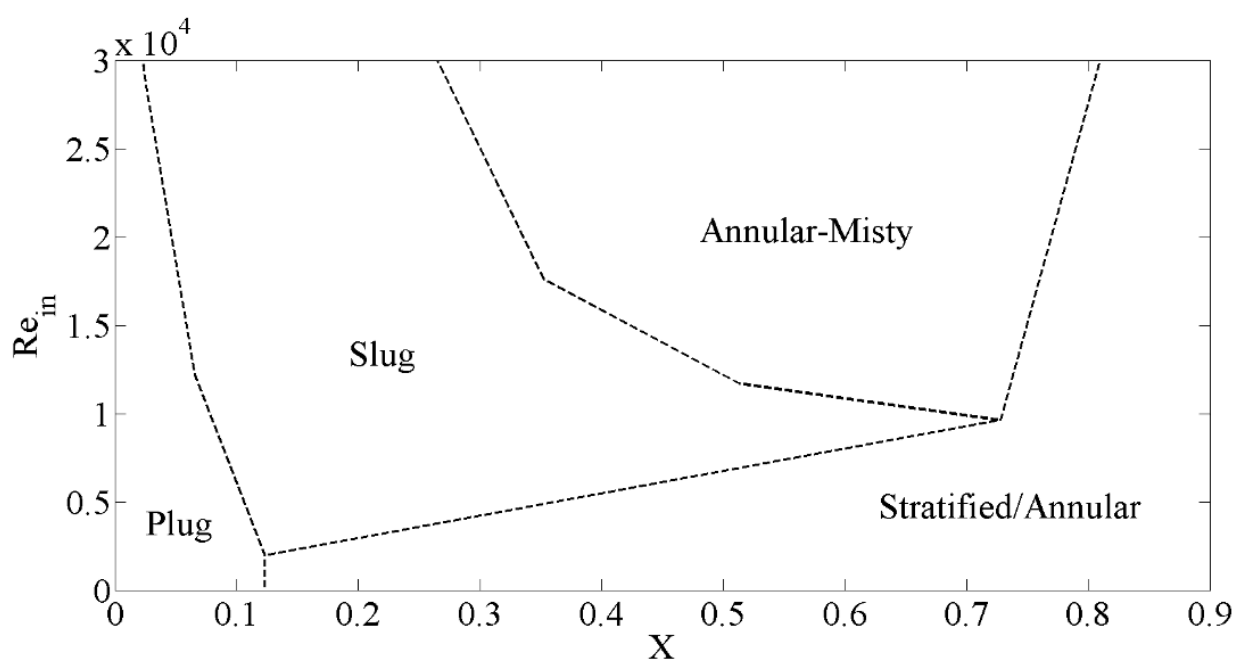

Figure 3.10: Close-up of the transformed Baker flow regime in the $\left\{\operatorname{Re}_{i n}, X\right\}$ domain to show the extents of the Stratified/Annular Regime. 
The Baker map transformed to the $\left\{R e_{i n}, X\right\}$ space in Figs. 3.9 and 3.10 is a lot easier to interpret directly without any additional calculations since it directly gives the vapor quality, $X$ at which transition occurs from flow regime to another for a given Reynolds number of the inlet vapor, $R e_{i n}$. There are again certain inconsistencies between the results reported by the Baker map and the experimental results observed in [2]. The transformed Baker map's results shown in Fig. 3.9 and 3.10 only report the Bubbly regime as being observed for higher $R e_{\text {in }}$ values $\left(R e_{i n} \geq 140000\right)$. However, as mentioned in section 3.3.1, for the experiments reported in [2], the bubbly regime was observed for even low values of $R e_{i n}$, in the range of 4000-17000.

The Baker map [8] in Fig 3.8 and its transformation in the $\left\{R e_{i n}, X\right\}$ space (Figs. $3.9,3.10)$ also show the presence of the misty-annular regime for low inlet vapor speeds (like the Taitel and Dukler map does in section 3.3.1). As mentioned earlier, misty-annular flow occurs a lot more easier in two phase flows with no phase change and its presence for low inlet Reynolds numbers for a map to be used for flow regime of internal condensing flows is not qualitatively accurate. This is again attributed to the likely focus of the Baker map largely on values of $R e_{\text {in }} \geq 100000$ and the fact that results reported are for experiments of gas/liquid flows and not for internal condensing flows. 
With some minor modifications, a qualitative modification of the Baker flow regime map in both its original axes values (Fig 3.11) and it's transformation to the $\left\{R e_{i n}, X\right\}$ space is shown (Fig. 3.12) below.

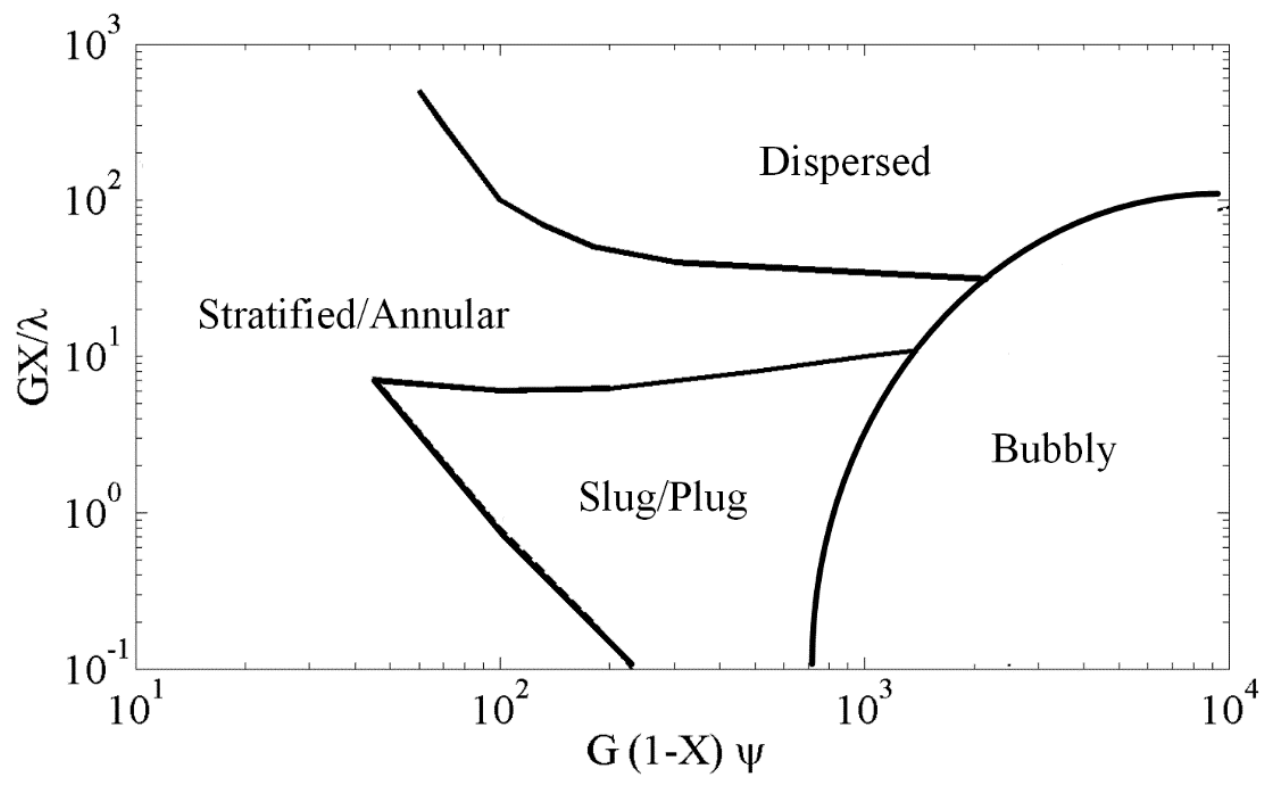

Figure 3.11: Schematic of the modified Baker Map for consistence with experimental results of [2] 


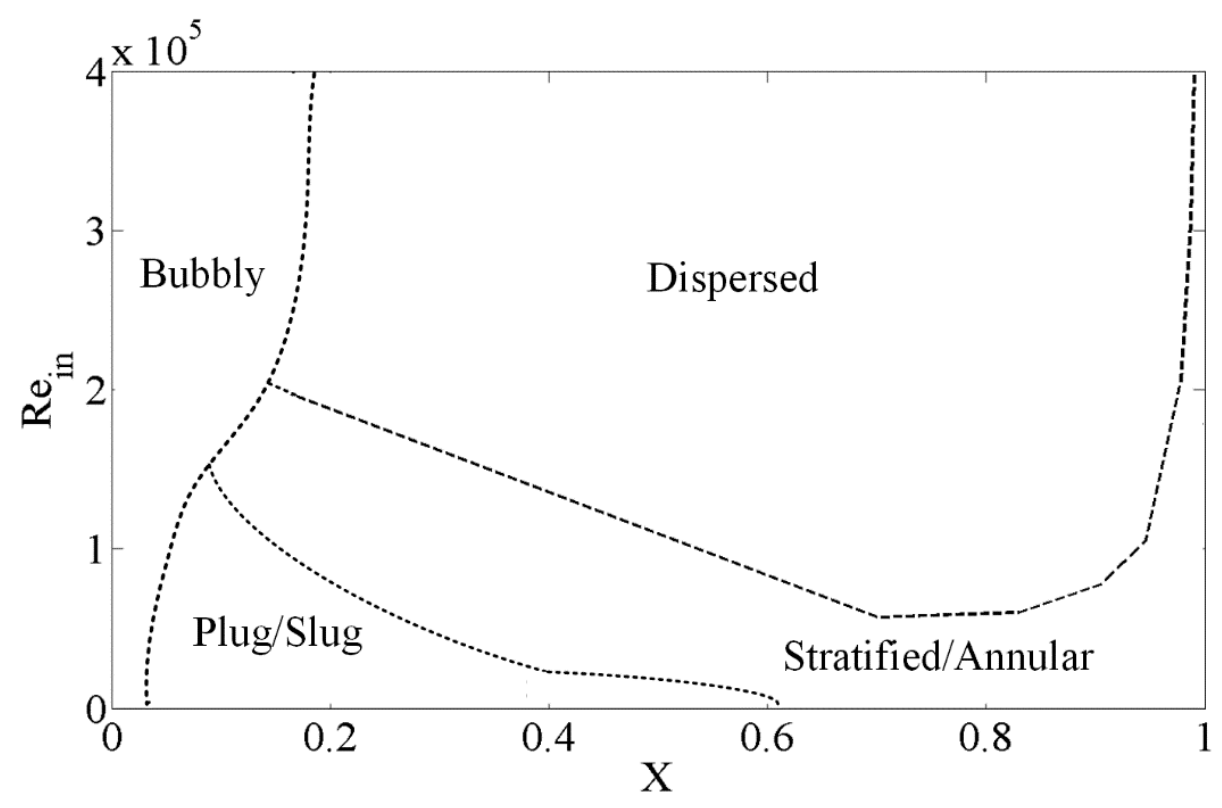

Figure 3.12: Transformed version of Fig. 3.11 in the $\left\{R e_{-} i n, X\right\}$ domain

\subsection{Relationship of Vapor Quality to Physical Distance along the Length of the Condenser}

One of the criticisms of this study regarding the status of current flow regime maps in literature is the fact that the transition curves in the flow regime maps are ostensibly independent of the thermal boundary conditions associated with internal condensing flows (or boiling flows). This is true because typical studies on flow regimes maps [7-12] believe that the use of vapor quality $X$ in the maps justifies neglecting the cooling (or heating) conditions modeled by the thermal boundary 
conditions (temperature controlled or heat-flux controlled). This is because the variation of $X$ with distance along the condenser length is a function of the thermal boundary condition imposed on the wall of the condenser. This variation of vapor quality as a function of physical distance is obtained from the energy balance equation for the control volume of the condenser. The energy balance equation, written for flow across an element of width $\Delta x$ (shown in Fig 2.1) gives an ordinary differential equation (ODE) for vapor quality which can be solved to make explicit the dependence of $X$ on physical distance $x$ (or $\hat{x}$ ). This energy balance is easily written as Eqs. 3.16 or 3.17 given below.

$$
\begin{aligned}
& \frac{d X}{d \hat{x}}=\frac{-q_{w}^{\prime \prime}(x)}{G h_{f g}} \equiv-q_{w-n d}^{\prime \prime} \mid \operatorname{avg} \psi(\hat{x}) \\
& \frac{d X}{d \hat{x}}=-\frac{\overline{J a}}{P r_{l}} \frac{N u_{x}}{R e_{\text {in }}} \frac{\mu_{2}}{\mu_{1}} \boldsymbol{\varphi}(\hat{x})
\end{aligned}
$$

In Eq. 3.16, $\left.q_{w-n d}^{\prime \prime}\right|_{\text {avg }}$ is the spatially average value of $\frac{q_{w}^{\prime \prime}(\hat{x})}{G h_{f g}}$ along the length of the condenser and $\boldsymbol{\psi}(\hat{x})$ is a non-dimensional function of $\hat{x}$ (similar to $\boldsymbol{\varphi}(\hat{x})$ ) which shows the variation of $\frac{q_{w}^{\prime \prime}(\hat{x})}{G h_{f g}}$ about its mean value of $q_{w-n d}^{\prime \prime} l_{\text {avg }}$. Eq. 3.16 is the equation to be used to obtain the variation of vapor quality with non-dimensional distance when the variation of the condensing surface's heat flux is known (or assumed). When the condenser's surface temperature variation is known (or assumed), Eq. 3.17 can be used to determine the variation of vapor quality with nondimensional distance along the condenser's length. 
Eqs. 3.16 and 3.17 are analytical equations that can be solved to obtain the variation of vapor quality with distance. However, for this approach, the empirical correlation for $h_{x}$ or $N u_{x}$ has to be available in the form given in Eq. 3.5. Alternatively, computationally obtained values of $h_{x}\left(\right.$ or $\left.q_{w}^{\prime \prime}(\hat{x})\right)$ can be used in Eq. 3.17 Another method that can be used to obtain this variation is by using the quasi $1 \mathrm{D}$ and $2 \mathrm{D}$ steady simulation tools mentioned in section 2 . Using the solutions to the condensing flow problem described in section 2 , the mass flux across the interface can be determined and thus the vapor and liquid mass flow rates can be determined along the length of the condenser giving rise to a computationally derived value of $X$. Fig. 3.9 shows the agreement between the variation of vapor quality with nondimensional distance as obtained by the solution of Eq. 3.16 and as obtained computationally using the quasi $1 \mathrm{D}$ simulation tool. This consistency is not surprising since Eq. 3.16 is obtained from the energy balance equation which is a part of the governing equations solved by both the quasi-1D and $2 \mathrm{D}$ steady simulation tools.

We can see from Fig. 3.13 that the variation of $X$ with $\hat{x}$ as obtained from both the solution of Eq. 3.16 and the solution from the quasi 1D simulation tool is the same as is expected.

Thus, in finding the relationship between the variation of the vapor quality with non-dimensional distance and the thermal boundary conditions on the condensing 
flow, either Eq. 3.16 can be solved or the computational solution can be used directly.

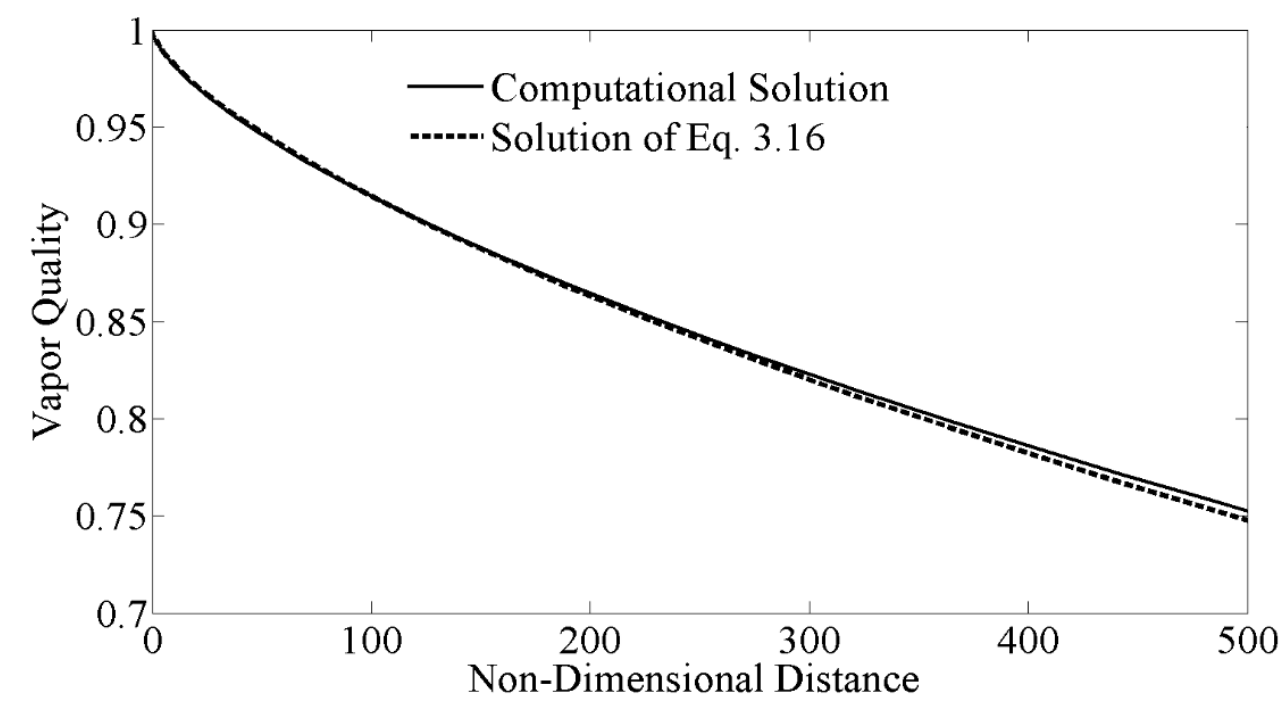

Figure 3.13: Agreement between the variation of vapor quality with nondimensional distance obtained from the quasi 1D simulation tool and the solution of Eq. 3.16.

3.4 A quantitative 3D Flow Regime Map in the $\left\{\operatorname{Re}_{i n}, \frac{J a}{P r_{l}}, \widehat{x}\right\}$ Space Obtained from a conversion of the Baker Flow Regime Map

As mentioned in section 3.2, a flow regime map in the $\left\{R e_{i n}, \frac{J a}{P r_{l}}, \hat{x}\right\}$ space depicts the transition between the different flow regimes in a very intuitive fashion - since 
these are the variables that can directly be computed through experimental measurements (see discussion following Fig. 3.1). Using the data from the modified schematic of the Baker map (Fig. 3.12) and the solution for the function $X(\hat{x})$, a flow regime map in the three dimensional space of $\left\{\frac{J a}{P r_{l}}, R e_{i n}, \hat{x}\right\}$ has been constructed. This construction is for the fluid R-134A at an inlet pressure of 100 KPa. Fig 3.14 shows one orientation of this 3D map.

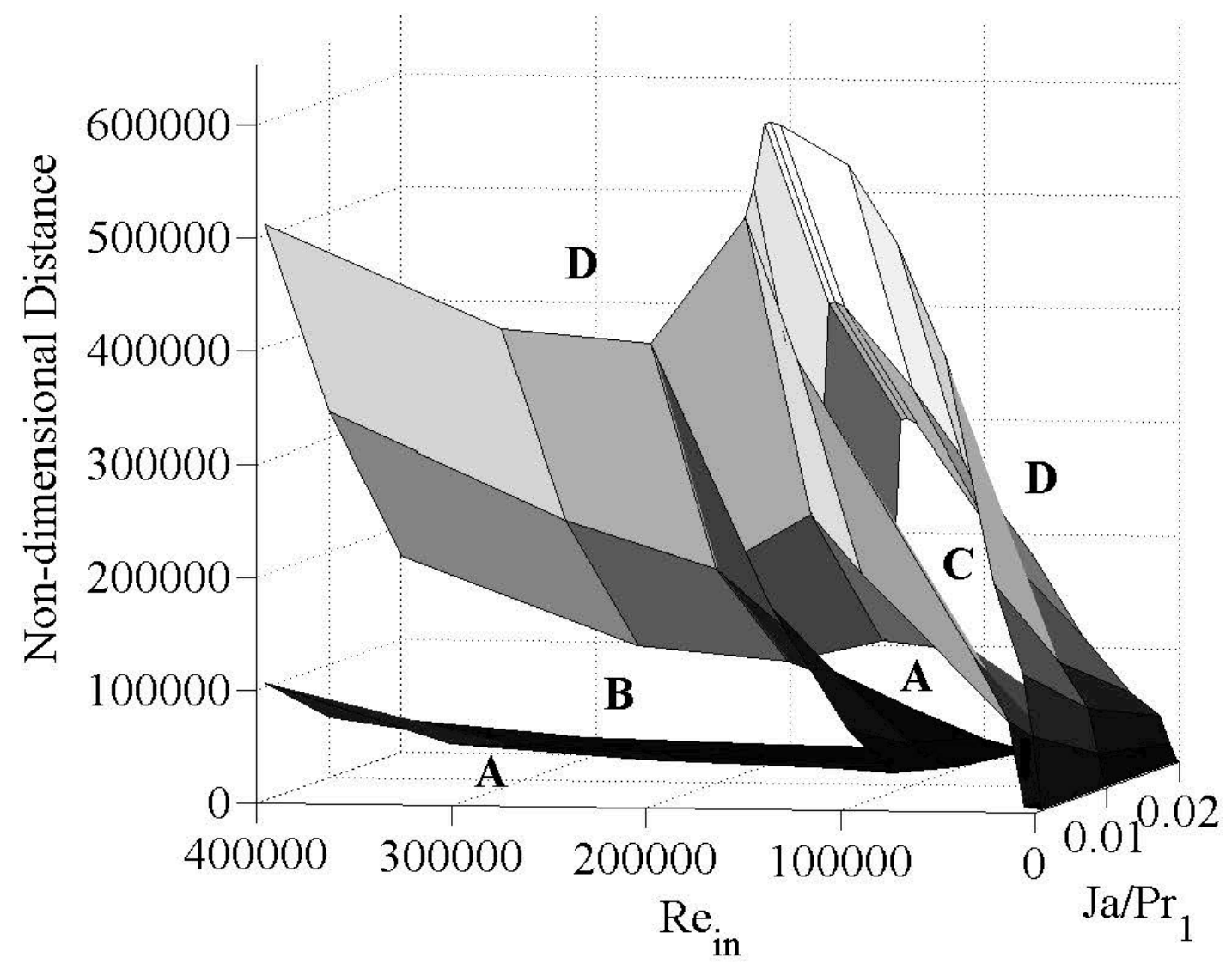

Figure 3.14: The different flow regimes shown in the $\left\{\frac{\mathrm{Ja}}{\mathrm{Pr} r_{l}}, R e_{i n}, \hat{x}\right\}$ domain obtained by a conversion of data from the modified Baker map in Fig. 3.12 for the fluid R- 
134A. The zones are labeled as: A - Stratified/Annular, B - Dispersed, C Slug/Plug, D - Bubbly

Fig. 3.14 incorporates the results from the Baker flow regime map [8] for higher $R e_{i n}$ values and the results observed in [2] for lower $R e_{i n}$ values in a single figure. This study expects that an accurate flow regime map in the three dimensional $\left\{R e_{i n}, \frac{J a}{P r_{l}}, \hat{x}\right\}$ space would qualitatively look similar to Fig. 3.14. It should be noted that also the dispersed flow regime is reported as separate from the stratified/annular regime in the Baker map [8], the difference between stratified/annular and the dispersed regime would be negligible at lower inlet Reynolds numbers. This is because the liquid film is more stable for two-phase flows with phase change when compared to gas-liquid flows for which the results in [8] are reported.

Although it is important to study the transition of all the different flow regimes in shear driven internal condensing flows, the flow regime of primary importance when it comes to the design of shear-driven flow condensers is the stratified/annular regime (A in Fig. 3.14). As mentioned in section 1.1, the goal of modern cooling systems is to be able to extract a high heat flux of the order of $1 \mathrm{~kW} / \mathrm{cm}^{2}$. In order to achieve this goal, extremely high heat transfer rates are required of modern sheardriven flow condensers. Thus the condensers need to be designed that they operate primarily in the stratified/annular regime since this is the flow regime that demonstrates the largest heat transfer rates due to the continuous presence of a thin liquid film along the heat transfer surface (as opposed to the presence of vapor 
bubbles on the surface with a much lower thermal conductivity). Simply the presence of the stratified/annular regime along the length of the condenser will not be sufficient for the high heat flux goal mentioned earlier. There are other ways by which the heat-transfer rates in the stratified/annular regime can be increased but that is beyond the focus of this study. It is touched upon in section 5 .

One of the primary goals of this study is to study the various engineering heat transfer correlations available in literature for the stratified/annular flow regime, examine their validity and to compare them with the heat-transfer correlation obtained by this study through a combination of the results from the quasi 1D simulation tool and the exact 2D steady simulation tool. This is described in section 4. 


\section{Heat Transfer in the Stratified/Annular Flow Regime of Shear-Driven Internal Condensing Flows}

\subsection{Engineering Heat Transfer Correlations in Literature}

As mentioned in section 3, most of the engineering heat transfer correlation presented in literature [14-18] neglect some of the parameters that one expects, from a first principles standpoint, to influence the heat transfer rates in a shear-driven flow condenser - particularly in the stratified/annular flow regime. This fact coupled with the fact that almost all heat transfer correlations published in literature are semi-empirical correlations causes some doubts about the general validity and accuracy of such correlations. This study aims to put a rest to this speculation by verify the underlying assumptions (such as Eq. 3.5) made by the correlations. It also points out the drawbacks of typical heat transfer correlations in literature and provides a heat transfer correlation obtained through the use of the computational tools mentioned in section 2. It then compares the results obtained from the proposed correlation to some of the popular heat transfer correlations in literature.

Before presenting the popular heat transfer correlations available in literature for shear-driven internal condensing flows, some parameters and symbols used in 
typical empirical heat transfer correlations need to be defined. These are defined in Eqs. $4.1-4.7$ below.

The turbulent-turbulent Lockhart-Martinelli parameter denoted by $X_{t t}$ is defined as:

$X_{t t}=\left(\frac{\mu_{1}}{\mu_{2}}\right)^{0.1}\left(\frac{1-X}{X}\right)^{0.9}\left(\frac{\rho_{2}}{\rho_{1}}\right)^{0.5}$

The two phase multiplier, $\phi_{g}$ is defined as:

$\phi_{g}=1+C X_{m}+X_{m}^{2}$

Here, $X_{m}$ is the Locakhart-Martinelli parameter as defined in Eq.3.3 and $C$ is the Lockhart-Martinelli coefficient defined in [14].

The vapor two-phase multiplier used in [17], denoted by $\phi_{V}$ is defined by:

$$
\phi_{V}=1+21\left(1-e^{-0.319 D_{h y d}}\right) X_{t t}+X_{t t}^{2}
$$

The liquid Reynolds number denoted by $R e_{1}$ is defined as:

$R e_{1}=\frac{G(1-X) D_{h y d}}{\mu_{1}}$

The vapor Reynolds number is similarly denoted by $R e_{2}$ and defined as:

$R e_{2}=\frac{G X D_{h y d}}{\mu_{2}}$

The "liquid-only" Reynolds number is denoted by $R e_{l o}$ and is defined by:

$R e_{l o}=\frac{G D_{h y d}}{\mu_{1}}$ 
The ratio of actual pressure to critical pressure in a channel or tube is denoted by $P_{R}$ and is defined as:

$P_{R}=\frac{P_{a c t}}{P_{c r i t}}$

\subsubsection{Kim and Mudawar Heat transfer correlation}

This correlation claims to be a universal correlation for obtaining the heat transfer coefficient for mini and micro-channel flows with a wide variation in operating fluids. It also provides heat transfer correlation for both the stratified/annular flow regime and the slug and bubbly flow regime. This study will however only focus on the stratified/annular flow regime since that is the primary flow regime of interest.

Eq. 4.8 describes the heat transfer correlation as provided by Kim and Mudawar [14].

$$
\frac{h_{x} D_{h y d}}{K_{1}}=0.048 \operatorname{Re}_{1}^{0.69} \operatorname{Pr}_{1}^{0.34}\left(\frac{\phi_{g}}{x_{t t}}\right)
$$

Clearly, this correlation does not explicitly show any dependence on the thermal boundary condition of the condensing flow. However, this equation provides the variation of the local heat transfer coefficient as a function of vapor quality because $\phi_{g}$ and $X_{t t}$ depend on $X$. In order to obtain the variation of the heat transfer coefficient with distance along the length of the condenser, Eq. 3.16 needs to be used with Eq. 4.8 . 
For a particular case of flow condensation with R134-A as the operating fluid, the variation of the heat transfer coefficient as a function of vapor quality is shown in Fig. 4.1

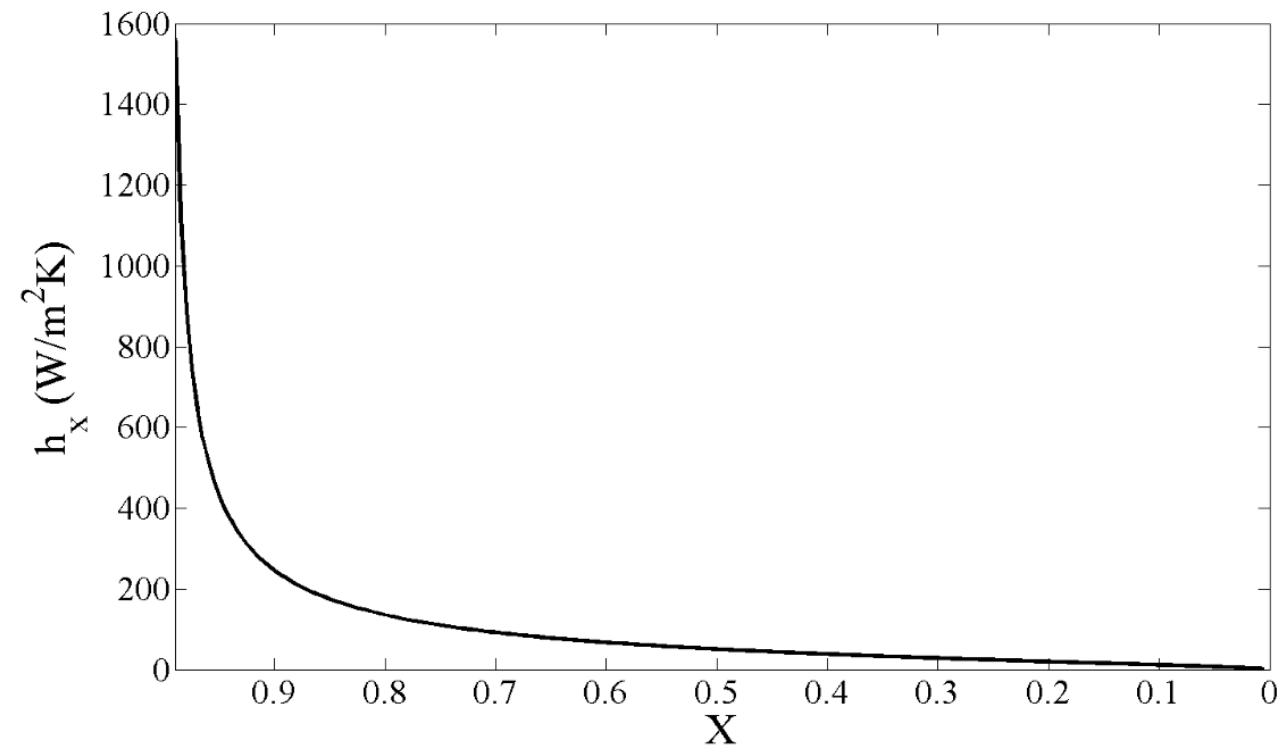

Figure 4.1: Variation of local heat transfer coefficient $h_{x}$ as a function of vapor quality $X$ obtained from Kim and Mudawar correlation [14] for fluid R-134A with inlet vapor speed $U_{\text {in }}$ of $1 \mathrm{~m} / \mathrm{s}$ and channel height of $0.002 \mathrm{~m}$

The particular case of shear-driven flow condensation for which the variation of $h_{x}$ is shown as a function $X$ is for inlet vapor speed $U_{\text {in }}$ of $1 \mathrm{~m} / \mathrm{s}$ and channel height of $0.002 \mathrm{~m}$. It should be noted here that the correlation is only for the value of the heat transfer coefficient in the stratified/annular flow regime. 


\subsubsection{Koyama et al. Heat Transfer Correlation}

This empirical correlation is similar to the Kim and Mudawar correlation [14] in that it does not explicitly show any dependence of the heat transfer coefficient on the thermal boundary condition associated with the condensing flow. The Koyama et al. correlation [17] however makes no mention of the flow regimes to which this correlation can be applied.

The equation presented by Koyama et al. to calculate the heat transfer coefficient is given by:

$\frac{h_{x} D_{h y d}}{K_{1}}=0.0152\left(1+0.6 P r_{1}^{0.8}\right)\left(\frac{\phi_{V}}{X_{t t}}\right) R e_{L}^{0.77}$

For the same case of shear-driven flow condensation of R-134A with inlet vapor speed $U_{\text {in }}$ of $1 \mathrm{~m} / \mathrm{s}$ and channel height of $0.002 \mathrm{~m}$, the variation of the local heat transfer coefficient with vapor quality is given in Fig. 4.2 


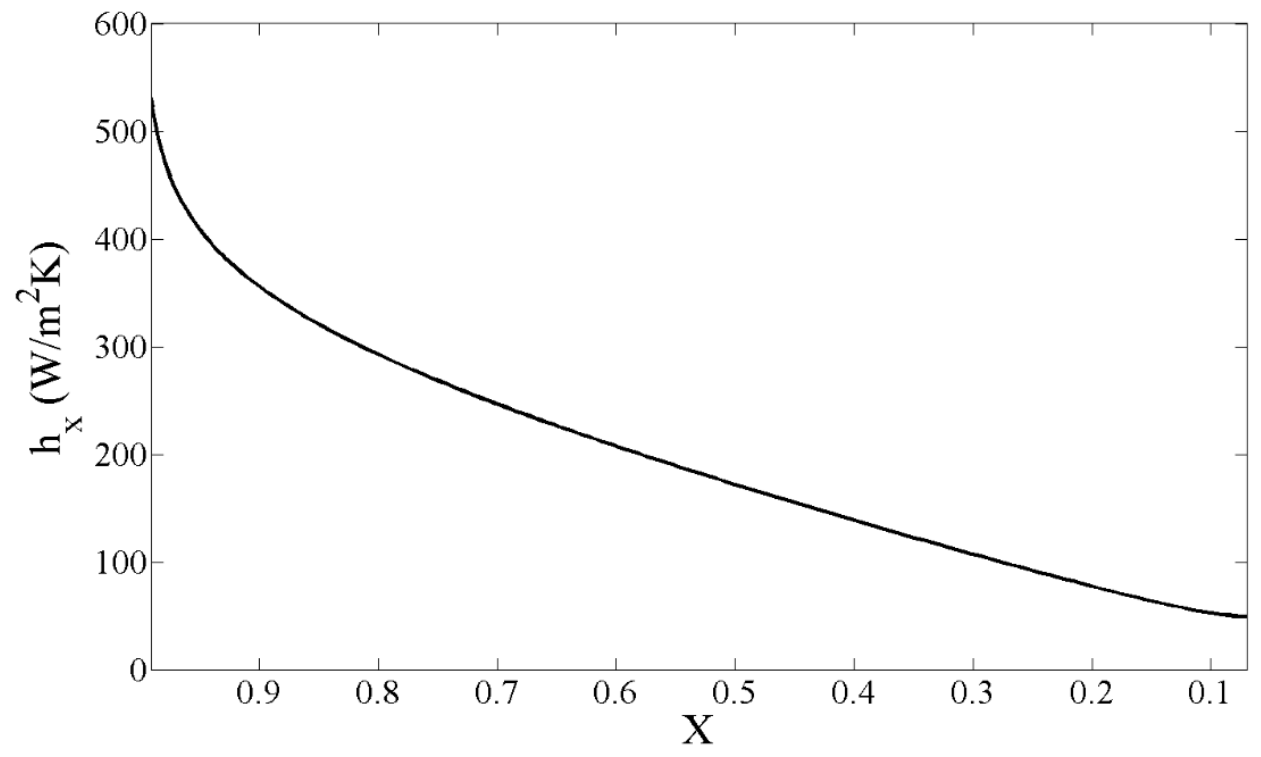

Figure 4.2: Variation of local heat transfer coefficient $h_{x}$ as a function of vapor quality $X$ obtained from Koyama et al. correlation [17] for fluid R-134A for inlet vapor speed $U_{\text {in }}$ of $1 \mathrm{~m} / \mathrm{s}$ and channel height of $0.002 \mathrm{~m}$

\subsubsection{Cavallini and Zecchin Heat Transfer Correlation}

The Cavallini and Zecchin correlation is similar in that there is no dependence of the heat transfer coefficient on the thermal boundary condition of the condensing flow. The equation describing this correlation is given in Eq. 4.10.

$\frac{h_{x} D_{h y d}}{K_{1}}=0.005 \operatorname{Pr}_{1}^{0.33}\left[\left\{R e_{2}\left(\frac{\mu_{2}}{\mu_{1}}\right)\left(\frac{\rho_{2}}{\rho_{1}}\right)^{0.5}\right\}+R e_{1}\right]^{0.8}$

For the same case of shear-driven flow condensation of R-134A with inlet vapor speed $U_{\text {in }}$ of $1 \mathrm{~m} / \mathrm{s}$ and channel height of $0.002 \mathrm{~m}$, the variation of the local heat transfer coefficient with vapor quality is given in Fig. 4.3 


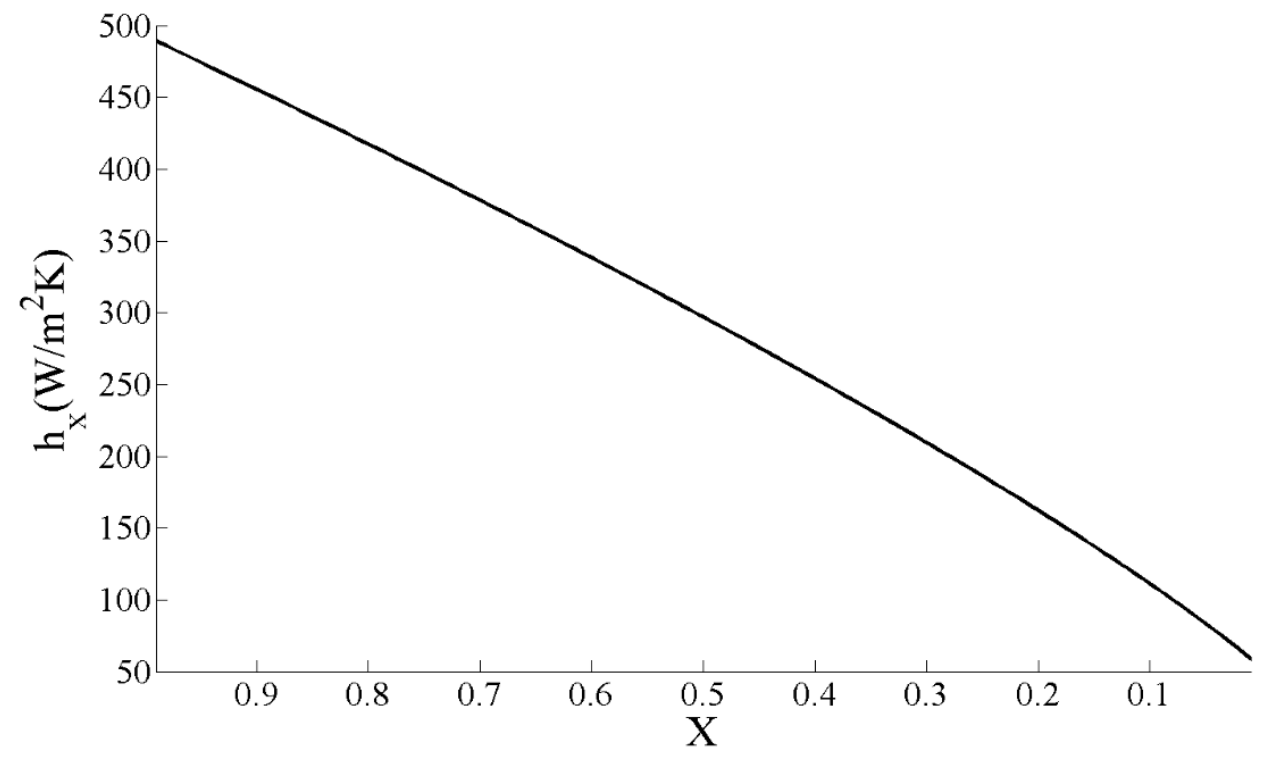

Figure 4.3: Variation of local heat transfer coefficient $h_{x}$ as a function of vapor quality $X$ obtained from Cavallini and Zecchin [16] correlation for fluid R-134A for inlet vapor speed $U_{-}$in of $1 \mathrm{~m} / \mathrm{s}$ and channel height of $0.002 \mathrm{~m}$

As can be observed from Figs. $4.1-4.3$, there is a noticeable difference in the results obtained from the three correlations. Also, some of the correlations do not specify the flow regime(s) to which they are applicable.

This study proposes its own correlation for the heat transfer coefficient in the stratified/annular regime of shear-driven internal condensing flows using computational solutions from the quasi-1D simulation tool and the exact $2 \mathrm{D}$ steady tool mentioned in section 2. 


\subsection{A Computationally Derived Heat Transfer Correlation for the Stratified/Annular Flow Regime of Shear-Driven Internal Condensing Flows}

This study proposes its own heat transfer correlation for the stratified/annular flow regime of shear-driven internal condensing flows that is derived from the results obtained from the quasi-1D simulation tool and the exact 2D steady simulation tool mentioned in section 2. Since both the simulation tools employ an interface tracking equation (to determine the boundary between the liquid and vapor phases) that assumes a continuous smooth interface, the results are valid only for the stratified/annular flow regime. Although this correlation applies only to the smooth interface subset of annular/stratified flows, it is well known [2] that a simple empirical multiplication factor of value $1.1-3$ should adequately model most of the annular/stratified regimes of operation. Also, the results (both in the original domain and the transformed domains) from the Baker flow regime map [8] described in section 3 have been used to determine the extents of the stratified/annular regime for each case whose results were employed in the generation of this correlation. With a subsequent improvement of flow regime maps and the ability of computationally determine flow regimes (see [23]), a better/sharper definition of the annular/stratified flow regime boundary is possible. 
Since the results obtained for the generation of this correlation come from the computational solution of the governing equations (section 2), the heat-transfer coefficient is obtained naturally as a function of the non-dimensional distance along the condenser length. These results are used to present a correlation that describes the variation of the local heat transfer coefficient as a function of the nondimensional distance along the condenser length.

In order to obtain a comparison with typical engineering heat transfer correlations available in literature, the results obtained from the solution of the governing equations were converted (using the computational method mentioned in section 2) to obtain the variation of the heat transfer coefficient with respect to the vapor quality. A correlation in this format has also been generated for a range of parameters.

Both correlations for the local heat transfer correlation have been presented as a function of non-dimensional parameters - this is for better generality. The range of values of the respective non-dimensional parameters are:

$0 \leq \hat{x} \leq \hat{x}_{A}$

$1 \geq X \geq X_{\text {cr }}$

$1000 \leq R e_{i n} \leq 30000$

$0.001 \leq \frac{J a}{P r_{1}} \leq 0.03$ 
$3.2 E-4 \leq \frac{\rho_{2}}{\rho_{1}} \leq 0.03$

$0.0113 \leq \frac{\mu_{2}}{\mu_{1}} \leq 0.06$

Eqs. 4.15 and 4.16 were obtained based on a range of real fluid values obtained by running cases for water, FC-72, R134-A, and R113. For each fluid several points were picked from the $\left\{R e_{i n}, \frac{J a}{P r_{1}}\right\}$ space with extents as defined by the ranges in Eqs. 4.13 and 4.14. For each run case, only the results that satisfied Eq. 4.11 for the correlation with non-dimensional distance and those that satisfied Eq. 4.12 for the correlation with vapor quality were used in generating the correlations.

4.2.1 Correlation as a function of distance along the length of the condenser

Based on several runs of the quasi-1D simulation tool and the exact 2D steady condensing flow solver, the following correlation was generated for the Nusselt number $\left(N u_{x}\right)$ as a function of the non-dimensional distance and several other key variables:

$\frac{h_{x} D_{h y d}}{K_{1}}=1.1108 \hat{x}^{-0.3312} \operatorname{Re}_{i n}{ }^{0.3626}\left(\frac{J a}{P r_{1}}\right)^{-0.3242}\left(\frac{\rho_{2}}{\rho_{1}}\right)^{-0.2380}\left(\frac{\mu_{2}}{\mu_{1}}\right)^{-0.5457}$

The values of $R^{2}$ that quantifies the goodness of fit for the correlation presented in Eq. 4.17 is 0.9192 . This would be qualified as a good fit for the amount of data that was obtained from several runs to come up with this correlation. The value of the 
error estimate for the fit is 0.0494 . This again qualifies the correlation as a great fit to the multiple runs used to generate the correlation. As stated earlier, to account for wave effects, a simple empirically adjustable factor (in the range of $1.1-3$ ) should multiple the right hand side of Eq. 4.17.

\subsubsection{Correlation as a function of vapor quality}

Similar to the correlation in Eq. 4.18, results from several runs of both computational tools were used to obtain the following correlation for the Nusselt number $\left(N u_{x}\right)$ as a function of the vapor quality and several other key variables:

$\frac{h_{x} D_{h y d}}{K_{1}}=9268.64 X^{3.725} \operatorname{Re}_{i n}{ }^{0.1294}\left(\frac{\rho_{2}}{\rho_{1}}\right)^{-0.2548}\left(\frac{\mu_{2}}{\mu_{1}}\right)^{-0.4856}$

The values of $R^{2}$ that quantifies the goodness of fit for the correlation presented in Eq. 4.18 is 0.8914 . This would be qualified as a fairly good fit for the amount of data that was obtained from several runs to come up with this correlation. The value of the error estimate for the fit is 0.0762 . This again qualifies the correlation as a great fit to the multiple runs used to generate the correlation.

\subsubsection{Comparison with existing engineering heat transfer correlations}

Fig. 4.3 shows the comparison between the results obtained from the correlation in Eq. 4.18 and the results of the correlations mentioned in section 4.1. 


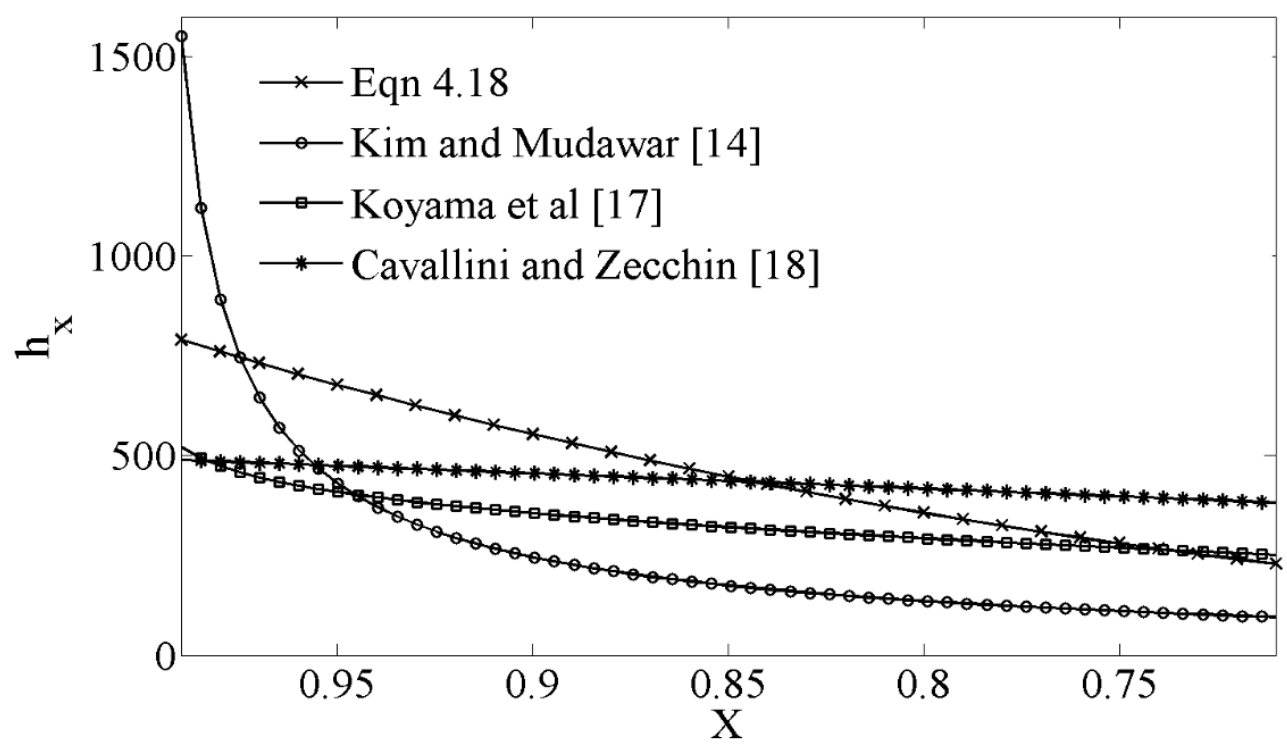

Figure 4.5: Comparison between the correlation obtained from this study (Eq. 4.18) and some popular engineering heat transfer correlations for flow condensation of R134A with inlet Reynolds number, $R e_{i n}=10000$

As is evident from Fig. 4.5, the correlation presented here in Eq. 4.18 compares well (within $\pm 15 \%$ for $X<0.95$ ) with the other correlations. This is encouraging since this is the only computationally derived correlation in the figure while all others are results from semi-empirical correlations.

It is important to note that for each fluid and inlet vapor speed, only the qualities that correspond to the stratified/annular regime, as defined by the transformed Baker map data (plotted in Fig. 3.2) were used to obtain the correlation. 


\section{Conclusions and Future Work}

\subsection{Conclusions with Regard to Flow Regime Maps}

Flow regime maps are extremely important to the study of Heat Transfer in Internal Condensing Flows because flow morphology determines the rate of heat transfer in the condenser. Stratified/annular flow regimes are of greater interest for modern flow condensers (see [3]) since the presence of a thin liquid film on the condensing surface enhances heat transfer rates significantly. Flow regime maps are of greater importance in shear-driven and horizontal channel flow condensers since the transition from stratified/annular to other thermally inefficient flow regimes typically occurs more easily (relative to gravity-driven flows) at higher qualities and shorter distances from the inlet of the condenser.

It is hence critical to estimate the qualities and distances at which transition occurs from one flow regime to another. Several flow regime maps in literature [7-12] address this critical need - but they often present their results in a language that is more suitable for gas-liquid flows as opposed to phase-change flows. Since the rate of heat and mass transfer has a significant effect on the transitions between flow 
regimes, there is a question as to whether the results from gas-liquid flows can be directly applied to the study of flow condensation.

When the flow regime maps presented in literature have no direct dependence on the thermal boundary condition applied, they are making the assumption that the value of the heat transfer correlation is known for each flow regime they are presenting, since the quality at which a certain flow regime transitions to another may not be the only critical parameter. Improvements to the typical flow regime maps in literature are needed through experiment-theory-computation synthesis rather than the current approach of an apriori assumed heat transfer correlation valid up to and beyond the critical transition zone.

Since the variation of vapor quality with distance along the length of the condenser is a function of the thermal boundary condition acting on the condenser (Eq. 3.17), the effect of thermal boundary condition is critical to be able to design flow condensers where the entire length of the condenser needs to be covered by the stratified/annular flow regime. Instead of this multi-step, convoluted approach of using currently available flow regime maps, this study makes a case for development of flow regime maps in the three-dimensional space of $\left\{\frac{J a}{P r_{1}}, R e_{i n}, \hat{x}\right\}$ by a direct experimental-theory-computation synthesis. The advantage of theory and experimental synthesis in estimating the transition between the stratified/annular and non-annular regimes is that the result obtained from such a study would be more general and trustworthy when validated against a few experimental cases. This is of 
importance since most flow regime maps typically conduct their experiments for a particular fluid, in a particular geometry and at a narrow range of inlet vapor speeds. Thus the quality and repeatability of the currently available estimates on $x_{A}$ are suspect across the entire range of parameters.

\subsection{Conclusions with Regard to Heat Transfer Coefficient}

A similar situation as the flow regime maps is observed with regard to the current state of knowledge for heat transfer correlations. Many heat transfer correlations pay no respect to the flow regime for which the correlation is presented and do not have sufficient experimental results to properly cover the parameter ranges for $\frac{J a}{\operatorname{Pr}_{1}}, \frac{\rho_{2}}{\rho_{1}}, \frac{\mu_{2}}{\mu_{1}}$, etc. This makes their results extremely suspect since the hydrodynamics of the two phase flow significantly affect the heat transfer process and will give rise to different heat transfer rates.

The most critical parameter ignored by typical heat transfer correlations is the thermal boundary condition of the condenser. Thermal boundary condition plays a significant role in determining the amount of heat transfer from the vapor and thus a correlation that does not take the thermal boundary condition into effect seems 
almost completely bogus - till one notes the indirect dependence of vapor quality on the thermal boundary condition.

The fact that the proposed heat transfer correlation (Eq. 4.18) is presented as a function of vapor quality is useful. But one cannot use this or other heat transfer correlations for heat transfer coefficient values to obtain the dependence of vapor quality with distance along the length of the condenser and to obtain the transition points in the flow regime maps. seems counter intuitive. However, having verified the typical heat transfer correlations presented as a function of vapor quality with the correlation obtained in this study by conversion of the variation with distance into a variation with vapor quality, this study has verified the basic approach (subject to improvements) and concurs with its validity.

The heat transfer correlation presented in this study is more trustworthy since it is based on a general exact computational solution that has been validated for several different cases with experimental results. 


\subsection{Future Work on Flow Regime Maps and Heat Transfer}

\section{Correlations}

A solution to many of the doubts associated with the impact of flow regime maps on the design of the flow condenser could be obtained if an accurate map of the kind shown in Fig. 3.12 is obtained. This is approximately done and is in the process of being refined by Dr. Amitabh Narain's research group at Michigan Technological University. The approximate ideas on how to theoretically arrive at such a flow regime map based on the flow physics is presented in [22-23]. Once this approach is refined and validated, it can be used as a universal flow regime map since the theory holds well for all fluids, flow rates and thermal boundary conditions.

The heat transfer correlation for the wavy stratified/annular regime is expected to be similar to that of the smooth stratified/annular flow regime with a constant multiplier in the range of $1.1-3$. This idea further needs to be extended and the constant multiplier would get much larger for pulsatile flows [31-34] where the inlet mass flow rate is fluctuated about a certain mean rate. One could expect the constant multiplier to then be in the range of $5-8$ (approximately based on experimental results in [31-34]).

The upper limit of the heat-flux enhancement remains to be reached by introducing resonance conditions within the condenser. The possibility of frequency matching 
between the interfacial waves, inlet mass flow rates and mechanical vibrations applied on the condensing surface needs to be investigated, since such large heat flux enhancements when observed with a high latent heat fluid such as water would essentially solve the modern heat flux removal requirements of $\sim 1 \mathrm{~kW} / \mathrm{cm}^{2}$. 


\section{References:}

1. Hill, S.A., Kostyk, C., Motil, B., Notardonato, W., Rickman, S., Swanson, T., "Thermal Management Systems Roadmap", Technology Area 14, National Research Council, National Aeronautics and Space Administration, November 2010

2. Kivisalu, M.T., Gorgitrattanagul, P., Narain, A., Results for High Heat Flux Flow Realizations in Innovative Operations of milli-meter Scale Condensers and Boilers, International Journal of Heat and Mass Transfer, 75, 381-398, 2014

3. Lasance, C.J., Simons R.E., "Advances In High-Performance Cooling for Electronics." Electronics Cooling, 11(4), 2005.

4. Wilson, J.R., "Electronics Cooling Depends on Innovative Approaches to Thermal Management", Military \& Aerospace Electronics, 2009.

5. Mitra, S., Narain, A., Naik, R., Kulkarni, S.D., A Quasi One-Dimensional Method and Results for Steady Annular/Stratified Shear and Gravity Driven Condensing Flows, International Journal of Heat and Mass Transfer, 54(15-16), 3761-3776, 2011.

6. Nusselt, W., "Die Oberflächenkondesation des Wasserdampfes", Z. Ver. Dt. Ing., 60(27), 541-546, 1916. 
7. Taitel, Y., Dukler, A.E., A Model for Predicting Flow Regime Transitions in Horizontal and Near-Horizontal Gas-Liquid Flow, AIChE Journal, 22(1), 47-55, January 1976.

8. Baker, O., Simultaneous Flow of Oil and Gas, Oil and Gas Journal, 53, 185-215, 1954.

9. Coleman, J.W., Garimella, S., Two-Phase Flow Regimes in Round, Square and Rectangular Tubes During Condensation of Refrigerant R134a, International Journal of Refrigeration, 26(1), 117-128, 2003

10. El Hajal, J., Thome, J.R., Cavallini, A., Condensation in Horizontal Tubes, Part 1: Two-Phase Flow Pattern Map, International Journal of Heat and Mass Transfer, 46(18), 3349-3363, 2003

11. Tandon, T.N., Varma, H.K., Gupta, C.P., A New Flow Regime Map for Condensation Inside Horizontal Tubes, Journal of Heat Transfer, 104.4, 763-768, 1982.

12. Traviss, D.P., Rohsenow, W.M., Flow Regimes in Horizontal Two-Phase Flow with Condensation, ASHRAE Transactions, 79.Part 2, 31-39, 1973.

13. Carey, V. P., Liquid-vapor phase-change phenomena, Taylor \& Francis, 2007 
14. Kim, S.M, Mudawar, I., Universal Approach to Predicting Heat Transfer Coefficient for Condensing Mini/Micro-Channel Flow, International Journal of Heat and Mass Transfer, 56.1, 238-250, 2013

15. Shah, M.M., A General Correlation for Heat Transfer During Film Condensation Inside Pipes, International Journal of Heat and Mass Transfer, 22, 547-556, 1979

16. Cavallini, A., Zecchin, R., A Dimensionless Correlation for Heat Transfer in Forced Convection Condensation, Proceedings Fifth International Heat Transfer Conference, 3, 309-313, Japan, 1974

17. Koyama, Shigeru, et al., An Experimental Study on Condensation of Refrigerant R134a in a Multi-Port Extruded Tube, International Journal of Refrigeration, 26(4), 425-432, 2003

18. Dobson, M. K., Chato, J. C., Condensation in Smooth Horizontal Tubes, Journal of Heat Transfer, Transactions ASME, 120(1), 193-213, 1998

19. Mitra, S., Development Of One-Dimensional And Two-Dimensional Computational Tools To Simulate Steady Internal Condensing Flows In Terrestrial And Zero-Gravity Environments, Doctor of Philosophy, Mechanical Engineering, Michigan Technological University, 2012 
20. Naik, R.R., Mitra, S., Narain, A., Shankar, N., Steady and Unsteady Computational Results of Full 2 Dimensional Governing Equations for Annular Internal Condensing Flows, COMSOL Conference Boston, 2013

21. Narain, A., Liang, Q., Yu, G., Wang, X., Direct Computational Simulations for Internal Condensing Flows and Results on Attainability/Stability of Steady Solutions, their Intrinsic Waviness, and their Noise-Sensitivity, Journal of Applied Mechanics, 71(1), 69-88, 2004

22. Naik, R.R., Narain, A., Mitra, S., Steady and Unsteady Simulations for Annular Internal Condensing Flows in a Channel, Submitted to Proceedings of the ASME 2014 International Mechanical Engineering Congress \& Exposition IMECE 2014, Montreal, Canada, November 2014

23. Naik, R.R., Development of Unsteady Two-Dimensional Computational Simulation Tools that Simulate Steady Annular Internal Condensing Flows and Characterize Interfacial Waves, Flow Stability and Flow Sensitivity, Ph. D., Michigan Technological University, Houghton, Michigan, 2014

24. Kulkarni, S. D., Narain, A., Mitra, S., Forced Flow of Vapor Condensing over a Horizontal Plate (Problem of Cess and Koh) - Steady and Unsteady Solutions of the Full 2D Governing Equations Journal of Heat Transfer, 132(10), 101502, 1-18, 2010 
25. Narain, A., Kulkarni, S. D., Mitra, S., Kurita, J. H., Kivisalu, M., Computational and Ground-based Experimental Investigations of the Effects of Specified and Unspecified (Free) Conditions at Exit for Condensing Flows in Terrestrial and Micro-gravity Environments, Annals of New York Academy of Sciences, Interdisciplinary Transport Phenomena in Space Sciences, 1161, 321-360, 2009

26. Mitra, S., Naik, R.R., and Narain, A., Numerical Simulation of Exact TwoDimensional Governing Equations for Internal Condensing Flows, COMSOL Conference Boston, 2011

27. Phan, L., Narain, A., Direct Computational Simulations that Yield Stability and Wave-Effects for the Classical Nusselt Problem of Film Condensation, IMECE2005-80221, Proceedings IMECE05 for Symposium on Gas Liquid and Phase Change Flows, Orlando, FL, USA, 2005

28. Dobran, F., Thorsen, R.S., Forced Flow Laminar Filmwise Condensation of a Pure Saturated Vapor in a Vertical Tube. International Journal of Heat and Mass Transfer, 23(2), 161-177, 1980

29. Narain, A., Modeling of Interfacial Shear For Gas Liquid Flows in Annular Film Condensation, Journal of Applied Mechanics, Transactions of the ASME, 63(2), $529-538,1996$ 
30. Chen, Y., Li, X., Wu, J., Shi, M., One Dimensional Numerical Simulation for Steady Annular Condensation Flow in Rectangular Microchannels, International Journal of Heat and Mass Transfer, 46(1), 75-82, 2009

31. Gorgitrattanagul, P., The length of the Annular Regime for Condensing Flows Inside a Horizontal Channel - The Experimental Determination of its Values and its Trends, Master of Science, Mechanical Engineering, Michigan Technological University, 2011

32. Kivisalu, M.T., Gorgitrattanagul, N., Narain, A., Naik. R., Hasan, M., Sensitivity of Shear-Driven Internal Condensing Flows to Pressure Fluctuations and its Utilization for Heat Flux Enhancements, International Journal of Heat and Mass Transfer, 56(1-2), 758-774, 2013

33. Kivisalu, M., Gorgitrattanagul, N., Mitra, S., Naik, R., Narain, A., Shear/Pressure Driven Internal Condensing Flows and their Sensitivity to Inlet Pressure Fluctuations. Published in Proceedings of IMECE ASME International Mechanical Engineering Congress and Exposition, Denver, Colorado, USA, 2011

34. Narain A., Kivisalu M., Gorgitrattanagul P., Naik R., Shankar N., Results for High Heat-flux Realizations in Innovative Operations of Milli-meter Scale Condensers and Boilers, Electronic Proceedings of the $22^{\text {nd }}$ National and $11^{\text {th }}$ International ISHMT-ASME Heat and Mass Transfer Conference, IIT Kharagpur, India, 2013 